\title{
Shockwave Treatment Enhances the Extracellular Matrix Production in Articular Chondrocyte Through Activation of the ROS/MAPK/Nrf2 Signaling Pathway.
}

\section{Po-Chih Shen}

Kaohsiung Medical University Chung Ho Memorial Hospital https://orcid.org/0000-0001-5185-5584

\section{Shih-Hsiang Chou}

Kaohsiung Medical University Chung Ho Memorial Hospital

Cheng-Chang Lu

Kaohsiung Medical University Chung Ho Memorial Hospital

Hsuan-Ti Huang

Kaohsiung Medical University Chung Ho Memorial Hospital

\section{Song-Hsiung Chien}

Kaohsiung Medical University Chung Ho Memorial Hospital

\section{Peng-Ju Huang}

Kaohsiung Medical University Chung Ho Memorial Hospital

\section{Zi-Miao Liu}

Kaohsiung Medical University Chung Ho Memorial Hospital

\section{Chia-Lung Shih}

Kaohsiung Medical University Chung Ho Memorial Hospital Department of Orthopaedic Surgery

\section{Shu-Jem Su}

Fooyin University

\section{Li-Min Chen}

E-DA hospital

Yin-Chun Tien ( $\sim$ d740113@kmu.edu.tw)

https://orcid.org/0000-0001-9069-6387

\section{Research}

Keywords: Shockwave, Osteoarthritis, Nrf2, Chondrocyte, Reactive oxygen species

Posted Date: June 8th, 2020

DOI: https://doi.org/10.21203/rs.3.rs-32232/v1 
License: (c) (i) This work is licensed under a Creative Commons Attribution 4.0 International License. Read Full License 


\section{Abstract}

Background: Shockwaves are acoustic pulses that have been widely used in the management of musculoskeletal disease. In current study, we investigated the biological effect of shockwaves on articular chondrocytes and clarified the mechanism by which chondrocytes sense and respond to the mechanical stimulation of shockwave treatment.

Methods: Porcine articular chondrocytes and human osteoarthritis (OA) chondrocytes were cultured in a three-dimensional pellet culture model and subjected to shockwaves. Then alamarBlue assay, Hoechest DNA assay, fluorescence-activated cell sorter, real-time polymerase chain reaction immunofluorescence staining, and western blotting were used to determine biological function and signaling transduction of chondrocytes after shockwaves.

Results: Shockwaves increased the sulfated glycosaminoglycan (GAG), Col2a1, and Acan production of chondrocytes without affecting cell viability and proliferation. Furthermore, shockwaves increased transient reactive oxygen species (ROS) production primarily through the action of xanthine oxidase and subsequently through phosphorylation of Erk1/2 and p38. Shockwaves also activated the nuclear translocation of Nrf2 and the Nrf2-related downstream gene expression of Ho-1 and Nqo-1. We suppressed the xanthine oxidase activity by allopurinol or specific siRNA, which significantly abrogated shockwave-induced mitogen-activated protein kinase (MAPK) phosphorylation, Nrf2 nuclear translocation, and extracellular matrix (ECM) synthesis. U0126 and SB203580, the specific inhibitors of MAPKs MEK1/2 and p38, respectively, significantly abolished shockwave-induced Nrf2 nuclear translocation and ECM synthesis. Inhibition of Nrf2 by both shRNA knockdown and brusatol reduced the shockwave-increased GAG, Col2a1, and Acan production.

Conclusions: Maintaining the homeostasis of the ECM of the articular cartilage is crucial for preventing the development OA. Our data indicate that shockwaves enhanced the ECM synthesis of chondrocytes through the activation of the ROS/MAPK/Nrf2 signaling axis. This study demonstrates that shockwaves holds promising therapeutic potential for $\mathrm{OA}$ and provides fundamental evidence for developing an optimal therapeutic strategy.

\section{Background}

Osteoarthritis (OA) is a leading cause of chronic disability and among the most important issues in the field of orthopedics. Patients with knee OA often have pain, deformity, and functional disability. The results achieved by conservative treatment for $\mathrm{OA}$ are not satisfactory, and the number of patients requiring joint arthroplasty surgery is increasing. With improved knowledge of early $O A$, novel regenerative approaches have recently gained greater attention. Shockwaves are acoustic pulses characterized by high positive pressure amplitudes and an extremely short buildup time. Emerging evidence suggests that shockwaves will potentially become a novel treatment for degenerative cartilage disease management and cartilage tissue engineering [1-5]. 
In animal studies and early clinical trials, shockwaves reduced OA symptoms, improved motor function, and had a beneficial biological effect on cartilage and subchondral bone $[1,6]$. Wang et al. demonstrated that applications of shockwaves have chondroprotective effects with decrease of articular cartilage degradation and regression of established knee OA changes induced by anterior cruciate ligament transection (ACLT) in rats[6-9]. According to Moretti et al., shockwaves might downregulate the expression of interleukin-10 (IL-10)[10]. They also found that tumor necrosis factor-a had no significant effect on chondrocyte proliferation, and revealed a dose-dependent increase in cytotoxicity in a human articular chondrocyte monolayer culture model. The biological effect of shockwaves on chondrocytes is not fully understood. The relevant results were obtained from the monolayer culture experimental design in which chondrocytes would undergo dedifferentiation and possibly lose their capacity to form cartilage, but these might not reflect the reality of cartilage undergoing shockwaves in joints[11]. To assess whether shockwaves have a therapeutic effect on OA, we applied shockwaves to chondrocytes in threedimensional (3D) culture model and investigated the safety, biological effect, and underlying molecular mechanism of shockwaves on articular chondrocytes.

Elevated production of reactive oxygen species (ROS) triggered by shockwaves was shown in tissue and cells, such as mesenchymal stem cells and adipose-derived stem cells[12-14]. ROS have been implicated in cellular damage. Excessive ROS can cause cellular damage and cell apoptosis, and they have long been implicated in the initiation of numerous diseases, including OA[15]. Recent studies have suggested that low levels of ROS can act as intracellular second messengers to regulate various crucial cellular activities, such as cell activation, proliferation, and death; furthermore, they can mediate the expression of numerous genes[16-18]. Chondrocytes produce ROS to resist stress in certain situations. The balance between ROS and intracellular antioxidant levels is important to maintaining the homeostasis of cartilage tissue and ensuring cell survival[15]. The transcription factor nuclear factor erythroid 2-related factor 2 (Nrf2), which controls several different antioxidant pathways, is an important regulator of cellular redox homeostasis[19]. Nrf2 regulates the gene expression of antioxidants such as superoxide dismutase 2 , NAD $(\mathrm{P}) \mathrm{H}$ :quinone oxidoreductase (Nqo-1), and stress response genes (e.g., heme oxygenase 1 [Ho-1]) through consensus cis-elements called antioxidant response elements (AREs). Recently, several studies have highlighted the pleiotropic roles of Nrf2 in preventing OA pathology, including as a regulator of chondrocyte apoptosis, inflammatory response, and cellular homeostasis[19-23]. Additionally, Nrf2 was considered as a mechanosensitive transcription factor in endothelial cells and bones[24]. On the basis of these reports, we hypothesized that the shockwaves induced ROS signals and that the subsequent AREs might be involved in the chondroprotective effect.

Shockwaves have been widely used for pain relief and the treatment of musculoskeletal disorders in recent years[5]. Use of shockwaves is noninvasive with a low complication rate, is feasible for outpatients, and has a relatively low cost compared with other conservative and surgical approaches $[5$, 25]. However, the chondroprotective effect of shockwaves on articular chondrocytes has not been fully elucidated, and the underlying mechanism is unknown[2]. Therefore, we investigated the biological effect of shockwaves on articular chondrocytes and clarified the mechanism by which chondrocytes sense and 
respond to the mechanical stimulation of shockwave treatment. Our studies aimed to fill this gap in knowledge by exploring the response and mechanism of shockwaves on cartilage.

\section{Methods}

\section{Reagents and antibodies}

Diphenyleneiodonium (DPI), allopurinol, rotenone, and dichlorofluorescein diacetate (DCFH-DA) were purchased from Sigma-Aldrich Co. (St. Louis, MO, USA). p38 inhibitor SB203580 was purchased from Calbiochem (San Diego, CA, USA). MEK1 and MEK2 inhibitor U0126 was obtained from Promega (Madison, WI, USA). Maxima First Strand cDNA Synthesis Kit, Lipofectamine 2000 reagent, and TRIzol RNA extraction kit reagent were purchased from Thermo Fisher Scientific (Waltham, MA, USA). Antibodies against aggrecan (Acan), xanthine oxidase (XO), Nrf2, p38, phospho-p38, and $\beta$-actin were purchased from Proteintech (Manchester, UK). Antibodies targeting phospho-JNK and JNK1/2/3 were purchased from MyBioSource.com. Antibodies against extracellular signal-regulated kinase 2 (Erk2) and phosphoErk1/2 were purchased from Cell Signaling Technology (Danvers, MA, USA). Anti-collagen type II and anticollagen type $X$ antibody were purchased from Arigo Biolaboratories Corp. (Arigo Bio, Hsinchu City, Taiwan, Republic of China). The small interfering RNA (siRNA) against porcine XO and the control siRNA were synthesized by TOOLS Biotechnology Co. (Taipei, Taiwan). The XO siRNA sequences are 5'GGAAGCAGAUAAUGUUGUUTT-3', and the negative control sequences are 5'-

UUCUCCGAACGUGUCACGUTT-3'. The small hairpin RNA (shRNA) of candidate genes and a luciferase control (pLKO.1-shluc) plasmid construct were obtained from the National RNAi Core Facility located at the Institute of Molecular Biology/Genomic Research Center, Academia Sinica (Taipei, Taiwan). The shRNA-targeted sequence was as follows: pLKO.1-Nrf2-shRNA targets the Nrf2 gene sequence 5'GCTCGGATCTACCTTCCAGTA-3'.

\section{Isolation and culture of porcine and human OA chondrocytes}

This study was approved by the Institutional Review Board and Institutional Animal Care and Use Committee of Kaohsiung Medical University Chung-Ho Memorial Hospital (KMUH-IRB-20140144). Articular chondrocytes used for primary culture were harvested from slices of distal femur cartilage from 8-week-old pigs and cartilage from patients with OA undergoing total knee replacement surgery. Chondrocytes were isolated by enzymatic digestion of $0.1 \%$ trypsin in phosphate-buffered saline (PBS) for $1 \mathrm{~h}$ and digested overnight with $0.01 \%(\mathrm{wt} / \mathrm{vol})(0.166 \mathrm{U} / \mathrm{mL})$ collagenase $\mathrm{P}$ (Boehringer/Roche, Mannheim, Germany) in medium in the presence of $10 \%$ fetal calf serum (FCS) at $37^{\circ} \mathrm{C}$ under agitation. After being collected by centrifugation, chondrocytes were resuspended in Dulbecco's modified Eagle's medium (DMEM)/F-12 supplemented with $10 \%$ fetal bovine serum and antibiotics $(100 \mathrm{U} / \mathrm{mL}$ penicillin and $100 \mathrm{U} / \mathrm{mL}$ streptomycin) and then seeded into a culture dish at $37{ }^{\circ} \mathrm{C}$ in humidified $5 \% \mathrm{CO}_{2}$ as a monolayer culture. The primary cells were subcultured to generation 2 for at least $24 \mathrm{~h}$ before different treatments. 
To form cell pellets, $5 \times 10^{5}$ cells were seeded per well in a round-bottomed $15-\mathrm{mL}$ tube in DMEM/F-12 condition medium and centrifuged for $5 \mathrm{~min}$ at $400 \times g$ as previously described [26]. Cell pellets were cultured for an additional $3 \mathrm{~d}$ before stimulation with or without shockwave treatment.

\section{Shockwave treatment}

In this study, shockwave treatment was performed with an electrohydraulic device (Duolith ${ }^{\circledR}$ SD1; Storz Medical AG, Trägerwilen, Switzerland). We used the chondrocyte pellet culture, a simple and effective model to evaluate the cell viability and molecular change of cell culture. The cell pellets were cultured for $5 \mathrm{~d}$ before the shockwave treatment to allow matrix to deposit around chondrocytes after pellet formation. The chondrocyte pellets were placed in a $10-\mathrm{mL}$ centrifuge tube, which was completely filled with medium to prevent air interference with impulse transmission, and then exposed to focused model shockwave applications using the extracorporeal shockwave system in a custom-made thermostatic acrylic container with a membrane to connect the shockwave applicator. Ultrasound transmission gel was used to couple this membrane to the applicator (Fig. 1a). A wedge-shaped absorber on the back wall was used to attenuate the interference by wave reflection. The acrylic container was filled with degassed water to avoid cavitation. A heater plate with a temperature sensor was connected to a control unit that allows the temperature to be regulated for imitation of in vivo conditions and to keep the cell cultures from cooling during the procedure. The focus of the shockwave was set at the bottom of the centrifuge tube, where the chondrocyte pellets were theoretically within the focal working zone of the shockwaves.

\section{alamarBlue assay for cell viability assessment}

For cell viability assessment, chondrocyte pellets or chondrocyte-agarose constructs were placed in $10 \%$ alamarBlue (Bio-Rad AbD Serotec Ltd., Kidlington, UK) and 90\% growth medium. The chondrocyte pellets or chondrocyte-agarose constructs were kept in an incubator in static culture for $24 \mathrm{~h}$. After $24 \mathrm{~h}$, the alamarBlue/growth medium mixture was collected and analyzed using Tecan GENios with Magellan 5 software (Tecan, Männedorf, Switzerland) to obtain absorbance readings at 570 and $600 \mathrm{~nm}$ for viability analyses. Percentage reduction of the alamarBlue, indicating proliferative activity, was determined from the absorbance readings using the standard manufacturer's protocols (Bio-Rad AbD Serotec Ltd.).

\section{Hoechst DNA assay for DNA content assessment}

DNA standard was prepared following the manufacturer's protocol. bisBenzimide H33258 solution $(2 \mu \mathrm{g} / \mathrm{mL})$ was prepared in $10 \times$ fluorescent assay buffer (Sigma-Aldrich), and $200 \mu \mathrm{L}$ of this was pipetted into a 96-well Nunc Fluor plate (Thermo Fisher Scientific). Samples and DNA standards $(10 \mu \mathrm{L})$ were then added to the H33258 solution. Samples were then read at 360-nm excitation and 460-nm emission at room temperature.

\section{Western blot analysis}

The cell lysates ( $40 \mu \mathrm{g}$ of protein for each) or nuclear extracts were prepared from vehicle-and shockwave-treated cells. An analytical $10 \%$ sodium dodecyl sulfate-polyacrylamide gel electrophoresis 
(SDS-PAGE) gel was prepared for Western blotting. After SDS-PAGE was executed, proteins were transferred to a polyvinylidene difluoride membrane using a semidry apparatus. We employed the following primary antibodies: porcine Acan; XO; Nrf2; p38; phospho-p38; phospho-JNK; JNK1/2/3; Erk; phospho-Erk; collagen, type II, a1 chain (Col2a1); and $\beta$-actin. Immunoblot analysis was carried out with mouse or rabbit immunoglobulin $\mathrm{G}(\mathrm{IgG})$ antibody coupled with horseradish peroxidase and detected using an enhanced chemiluminescence kit (MilliporeSigma, Burlington, MA, USA). The density of the immunoblots was determined using an image analysis system installed with BIO-ID software (Vilber Lourmat, Marne-la-Vallée, France).

\section{Cytosolic and nuclear extract preparation}

Chondrocytes on dishes were washed twice with PBS and scraped in $500 \mu \mathrm{L}$ of PBS. The cells were collected by centrifuging at 12,000 rpm for $10 \mathrm{~s}$, resuspended in $400 \mu \mathrm{L}$ of buffer $\mathrm{A}$ [10 mM 4-(2hydroxyethyl)-1-piperazineethanesulfonic acid [HEPES] [pH 7.9], $1.5 \mathrm{mM} \mathrm{MgCl}_{2}$, and $10 \mathrm{mM} \mathrm{KCl]}$, and then stored on ice for $10 \mathrm{~min}$. Nuclei were precipitated by centrifuging at $12,000 \mathrm{rpm}$ for $10 \mathrm{~s}$. The supernatants were extracted from the cytosol and harvested. The pellets were resuspended in $100 \mu \mathrm{L}$ of buffer $\mathrm{C}$ (20 mM HEPES [pH 7.9], $1.5 \mathrm{mM} \mathrm{MgCl}_{2}, 0.2 \mathrm{mM}$ ethylenediaminetetraacetic acid [EDTA], $420 \mathrm{mM}$ $\mathrm{NaCl}$, and $25 \%$ [vol/vol] glycerol) and then stored on ice for $20 \mathrm{~min}$. The suspension was centrifuged at $12,000 \mathrm{rpm}$ for $2 \mathrm{~min}$, and supernatants were collected and stored at $-70^{\circ} \mathrm{C}$ until use. Buffer $\mathrm{A}$ and buffer C contained $0.5 \mathrm{mM}$ dithiothreitol (DTT), $2 \mu \mathrm{g} / \mathrm{mL}$ leupeptin, $1 \mathrm{mM}$ orthovanadate, $2 \mu \mathrm{g} / \mathrm{mL}$ pepstatin A, and $0.5 \mathrm{mM}$ phenylmethylsulfonyl fluoride.

\section{RNA isolation and real-time polymerase chain reaction}

Total RNA was extracted from chondrocytes with and without shockwave treatment using TRIzol reagent (ThermoFisher). Total RNA $(2 \mu \mathrm{g})$ was reverse-transcribed into cDNA using the Maxima First Strand cDNA Synthesis Kit (ThermoFisher) according to the manufacturer's instructions. Briefly, the solution was incubated at $65^{\circ} \mathrm{C}$ for $5 \mathrm{~min}$; it was mixed with first-strand buffer, DTT, and RNaseOUT (ThermoFisher) for a final volume of $20 \mu \mathrm{L}$. Then, the solution was incubated at $42^{\circ} \mathrm{C}$ for $60 \mathrm{~min}$ and at $70^{\circ} \mathrm{C}$ for $15 \mathrm{~min}$ to inactivate the reverse transcriptase activity. Real-time polymerase chain reaction (PCR) was conducted using SYBR Green PCR Master Mix (Qiagen, Carpinteria, CA, USA) and was processed on a LightCycler PCR and detection system (Roche Diagnostics, Basel, Switzerland). Each reaction $(20 \mu \mathrm{L})$ was run in duplicate along with the following primer sequences: Col2a1, forward (ACTCCT GGCACGGATGGTC) and reverse (CTTTCTCACCAACATCGCCC); Acan, forward (CCCAACCAGCCTGACAACTT) and reverse (CCTTCTCGTGCCAGATCATCA); collagen, type $X$, a1 chain (Col10a1), forward (TGAACTTGGTTCATGGAGTGTTTTA) and reverse (TGCCTTGGTGTTGGATGGT); matrix metallopeptidase 13 (MMP13), forward (ACCCAGGAGCCCTCATGTTTCC) and reverse (CAGGGTTTCTCCTCGGAGACTG); Ho-1, forward (GGTCCTCGAAGAAGCCAAGACC) and reverse (GACCGTTGCCACCAGAAA GCTG); Nqo-1, forward (CTGCCATGTATGACAAGGGACC) and reverse (AGTGTGCCC AATGCTGTACGTC); and glyceraldehyde 3-phosphate dehydrogenase (GAPDH), forward (TCACGACCATGGAGAAGGCT) and reverse (CAGGAGGCATTGCTGATGATC). Cycling parameters were as follows: $95^{\circ} \mathrm{C}$ for $15 \mathrm{~min}$ to activate DNA polymerase, followed by 40 cycles of $95^{\circ} \mathrm{C}$ for $15 \mathrm{~s}, 60^{\circ} \mathrm{C}$ for $20 \mathrm{~s}$, and $72{ }^{\circ} \mathrm{C}$ for $30 \mathrm{~s}$. Melting 
curves were generated at the end of the reaction. Threshold cycles $\left(C_{t}\right)$ for each gene tested were normalized to the housekeeping $G A P D H$ gene value $\left(\Delta \mathrm{C}_{t}\right)$, and every experimental sample was normalized to its control $\left(\Delta \Delta \mathrm{C}_{\mathrm{t}}\right)$. Fold change values were expressed as $2^{-} \Delta \Delta \mathrm{Ct}$.

\section{Detection of ROS production}

The ROS production was detected as described previously, with slight modifications[27]. Chondrocytes with and without shockwave treatment were incubated with $20 \mu \mathrm{M} \mathrm{DCFH-DA} \mathrm{in} \mathrm{DMEM} \mathrm{for} 30 \mathrm{~min}$ at $37^{\circ} \mathrm{C}$ in the dark. The nonfluorescent DCFH can be oxidized by intracellular ROS to highly fluorescent dichlorofluorescein (DCF). For the quantification of ROS, cells were trypsinized and washed with ice-cold PBS to quantify the fluorescence emitted from the fluorescent DCF using a fluorescence-activated cell sorter (FACScan, BD Biosciences).

\section{Transient transfection}

The transfection was performed using Lipofectamine 2000 reagent according to the manufacturer's instructions with a slight modification. Chondrocytes were subcultured at a density of $6 \times 10^{5}$ cells in a 6$\mathrm{cm}$ dish and grown to $70-80 \%$ confluence before transfection. Then, the cells were transfected with XO siRNA or a negative control siRNA. siRNA was mixed with Lipofectamine 2000 reagent in $1 \mathrm{~mL}$ of OptiMEM (ThermoFisher) and then incubated with cells at room temperature $\left(25^{\circ} \mathrm{C}\right)$ for $30 \mathrm{~min}$. Cells were incubated in the mixture at $37^{\circ} \mathrm{C}$ for $48 \mathrm{~h}$. Then, the cells were harvested and pelleted in the complete medium. The cell pellets were given the optimal intensity of shockwave treatment, as described previously. After shockwave treatment, cells were incubated for an additional time period. The effects of siRNAs on the expression of Col2a1, Acan, and sulfated glycosaminoglycan (GAG) and its downstream signal pathway proteins were assessed using Western blotting and 1,9-dimethylmethylene blue (DMMB) assay.

\section{Immunofluorescence and histological analysis}

Chondrocytes cultured on coverslips or pellet tissues were fixed with $4 \%$ paraformaldehyde for 30 min (PBS, $0.1 \%$ Triton $X-100$, and $1 \%$ bovine serum albumin [BSA]), then permeabilized with $1 \%$ Triton $X-100$ for $1 \mathrm{~min}$. The samples were then incubated with blocked 1\% BSA for $20 \mathrm{~min}$ and antibodies against Nrf2, Col2a1, and Col10a1 overnight at $4{ }^{\circ} \mathrm{C}$. Samples were washed thrice in PBS and then incubated with FITC 488 goat antirabbit IgG or TRITC 543 goat antirabbit IgG for $1 \mathrm{~h}$ at room temperature. Samples were washed and mounted with Fluoroshield with 4',6-diamidino-2-phenylindole (Sigma-Aldrich) and visualized on an Olympus IX81 inverted fluorescence microscope (Olympus Microscopy, Tokyo, Japan). For histological analysis, pellet tissues were fixed in $10 \%$ buffered formaldehyde for $4 \mathrm{~h}$, then frozensectioned at a thickness of $8 \mu \mathrm{m}$. Alcian blue was used to detect the GAG. The staining intensity was compared between the two chondrocyte groups using a light microscope.

\section{Total GAG quantification and DNA content assays}

Total sulfated GAG content was determined in pellet samples and culture supernatant using DMMB (Polysciences, Warrington, PA, USA). Chondroitin sulfate $\mathrm{C}$ from shark cartilage was used as a standard. 
Briefly, cell pellets were digested with proteinase $\mathrm{K}$ solution $(1 \mathrm{mg} / \mathrm{mL}$ proteinase $\mathrm{K}$ in Tris-EDTA buffer [pH 7.6]) for $16 \mathrm{~h}$ at $65^{\circ} \mathrm{C}$. The digested sample $(100 \mu \mathrm{L})$ was then combined with $1 \mathrm{~mL}$ of dimethylmethylene blue dye solution, and absorbance was immediately measured at $656 \mathrm{~nm}$. DNA was measured using Hoechst 33258 dye. Briefly, $10 \mu \mathrm{L}$ of the digested sample was combined with $200 \mu \mathrm{L}$ of Hoechst dye solution $(0.7 \mu \mathrm{g} / \mathrm{mL})$. Fluorescence measurements were taken with an excitation wavelength of $340 \mathrm{~nm}$ and an emission wavelength of $465 \mathrm{~nm}$. A standard curve was obtained from calf thymus DNA. GAG content was normalized to the amount of DNA measured per sample and expressed as micrograms of $\mathrm{GAG} /$ microgram of DNA.

\section{Stable Nrf2-silencing articular chondrocyte transfectants}

293T cells $\left(8 \times 10^{6}\right)$ were incubated on $10-\mathrm{cm}$ petri dishes coated with poly-l-lysine for $24 \mathrm{~h}$ to package lentiviral vectors. TransIT-LT1 reagent (45 $\mathrm{LL}$; Mirus Bio, Madison, WI, USA) in Opti-MEM (750 $\mu \mathrm{L})$ was mixed with the packaging vector PCMV- $\triangle \mathrm{R} 8.91(6.75 \mu \mathrm{g})$, the envelope vector pMD.G $(0.75 \mu \mathrm{g})$, and the transfer vector pLKO.1-Nrf2-shRNA $(7.5 \mu \mathrm{g})$ or pLK0.1-shluc $(7.5 \mu \mathrm{g})$. After incubation at room temperature for $20 \mathrm{~min}$, the plasmid-containing mixture was transferred to the $293 \mathrm{~T}$ cells for $16 \mathrm{~h}$. After $1 \%$ (wt/vol) FBS-containing medium was added for $2 \mathrm{~d}$, the supernatant of the culture cells was harvested. The media containing lentiviral vectors were centrifuged at $2500 \mathrm{rpm}$ for $10 \mathrm{~min}$, and the viral supernatants were passed through $0.45-\mu \mathrm{m}$ filters. Primary chondrocytes (P2) were cultured on 6-cm petri dishes with $4 \mathrm{~mL}$ of medium, followed by infection with the harvested lentiviral supernatants $(1 \mathrm{~mL})$ and Polybrene $(8 \mu \mathrm{g} / \mathrm{mL})$ for $24 \mathrm{~h}$. The infected cells were incubated in $2 \mu \mathrm{g} / \mathrm{mL}$ puromycin-containing medium for the selection of stable Nrf2-silencing chondrocyte transfectants.

\section{Results}

\section{Shockwaves did not affect the viability and cell proliferation of porcine chondrocytes}

We first examined the effect of shockwaves on the viability of porcine chondrocytes. Cell pellets were treated with 100 and 500 shockwave pulses at energy levels of $0.07,0.15$, or $0.3 \mathrm{~mJ} / \mathrm{mm}^{2}$ thrice per week. The cell viability was measured with the alamarBlue test after the first, second, and third shockwave treatments and $7 \mathrm{~d}$ after shockwave treatment. The viability of chondrocyte pellets subjected to shockwaves with an energy level $\leq 0.3 \mathrm{~mJ} / \mathrm{mm}^{2}$ or $\leq 500$ impulses remained at $>95 \%$ that of untreated controls through the entire experimental period (Fig. 1b). Total cell numbers and the cell growth of chondrocyte pellets were measured (DNA quantification) at the end of the experiments. No significant difference was noted in the total DNA amount of chondrocyte pellets between the control and any shockwave treatment group (Fig. 1c).

\section{Shockwaves enhanced the ECM synthesis of porcine chondrocytes}


The synthesis of GAG is an important hallmark of chondrogenic differentiation. The porcine chondrocyte pellets were subjected to shockwaves with indicated energy and impulses thrice within $1 \mathrm{wk}$ and cultured for an additional $7 \mathrm{~d}$. The GAGs released into the in-culture supernatants and pellets were collected and quantified by DMMB assay and normalized by the DNA amount per pellet. Compared with the control group, the total amount of GAG/DNA was significantly elevated after shockwave treatment, except when the energy level was $0.07 \mathrm{~mJ} / \mathrm{mm}^{2}$ and the impulse was 500 (control vs. $0.07 \times 500,1.51$ times, $p<.05$; control vs. $0.15 \times 100,2.26$ times, $p<.001$; control vs. $0.15 \times 500,1.97$ times, $p<.001$; control vs. $0.30 \times$ $100,1.95$ times, $p<.001$; control vs. $0.30 \times 500,1.70$ times, $p<.01$ ) (Fig. $1 \mathrm{~d}$ ).

The expression of the Col2a 1 and Acan genes peaked at $24 \mathrm{~h}$ after shockwave treatment (Col2a1, control vs. $0.15 \times 500,2.06$ times, $p<.01$; Acan, control vs. $0.15 \times 500,1.39$ times, $p<.001$ ) (Fig. $1 \mathrm{e}$-f). But the mRNA expression of MMP13 and Col10a1, markers of hypertrophic chondrocytes, were not significantly elevated after shockwave treatment (Fig. 1g-h). Western blotting confirmed the gene expression data. The time-series Western blot study revealed that the protein expression of Col2a 1 and Acan were gradually elevated and peaked at $24 \mathrm{~h}$ after shockwave treatment (Col2a1, control vs. $0.15 \times 500,2.54$ times, $p$ $<.001$; Acan, control vs. $0.15 \times 500,2.33$ times, $p<.001$; Fig. 1i). However, the protein expression of Col10a1 and MMP13 was not increased after shockwave treatment. Col2a1 and Acan protein amounts were increased after shockwave treatment in a dose-dependent manner (Fig. 1j).

\section{Shockwaves increased the intracellular ROS production of porcine chondrocyte in a dose- and time-dependent manner}

Previous studies have demonstrated that shockwave treatment could affect cell function through ROS production[12,13]. In our study, a significant elevation in ROS production was observed 10 min after shockwave treatment (control vs. $0.15 \times 500,1.41$ times, $p<.01$; Fig. 2a). The shockwave-induced ROS production returned to basal levels 30 min later. The increase of ROS production activated by shockwaves was in a dose-dependent manner (control vs. $0.03 \times 500,1.21$ times, $p<.001$; control vs. $0.07 \times 500,1.40$ times, $p<.001$; control vs. $0.15 \times 500,1.64$ times, $p<.001$; Fig. $2 b$ ).

Superoxide $\left(\mathrm{O}_{2} \cdot\right)$ and hydrogen peroxide $\left(\mathrm{H}_{2} \mathrm{O}_{2}\right)$ are two of the most important ROS participating in cartilage homeostasis, aging, and OA[28]. To identify whether shockwave-induced ROS are produced by mitochondria, NADPH oxidase, or XO, we examined the effects of rotenone (inhibitor of mitochondria complex I), DPI (NADPH oxidase inhibitor), and allopurinol (XO inhibitor) on shockwave-induced ROS production. In the present study, the production of ROS following shockwave treatment was significantly attenuated by allopurinol but not by other inhibitors (Fig. 2c). This suggests that XO was responsible for shockwave-augmented $\mathrm{O}_{2}$. production.

Shockwaves induced mitogen-activated protein kinase signaling in porcine chondrocytes

Mitogen-activated protein kinases (MAPKs) are a family of protein kinases that mediate signal transduction from extracellular stimulation to the nucleus[29]. Previous studies have suggested that shockwaves can produce mechanical stimulation through pressure change and subsequently initiate 
serial cellular function by triggering various intracellular signaling events that involve MAPK signaling[12, 30]. Therefore, we examined whether the shockwave-induced chondrogenic effect is induced through the activation of MAPKs by analyzing the phosphorylation levels of Erk, JNK, and p38. The time-dependent phosphorylation of Erk, JNK, and p38 MAPK was monitored following shockwave treatment (Fig. 2d). A significant increase in the phosphorylation of both p38 MAPK and Erk1/2 after shockwave treatment was evident. Our results illustrate that the stimulation of porcine chondrocyte pellets with shockwaves activates the phosphorylation of Erk1/2 and p38 within 60 min. However, the phosphorylation of JNK was not elevated after shockwave treatment.

\section{ROS production generated from XO regulated shockwave-promoted subsequent activation of MAPK signaling and extracellular matrix (ECM) synthesis in porcine chondrocytes}

Previous studies have revealed that MAPK signaling could be activated by ROS in various cell types[21, 29]. To test whether the increased phosphorylation of Erk $1 / 2$ and p38 is dependent on shockwaveinduced ROS production, the chondrocyte pellets were pretreated with allopurinol before shockwave treatment. We found that the shockwaves elevated the phosphorylation of Erk1/2 and p38 (Fig. 3a-b), mRNA and protein expression of Col2a1, and Acan (Fig. 3c-e) and GAG production (Fig. 3f), all of which returned to the baseline level after treatment with allopurinol. To further confirm and better describe the role of XO in shockwave-induced production of ROS, the porcine chondrocytes were transfected with siRNA specific for XO using nucleofection. siRNA-mediated depletion of XO significantly abrogated the shockwave-induced phosphorylation of Erk1/2 and elevated protein production of Col2a1 and Acan (Fig. 3g-h). Similar results were also found in shockwave-induced elevated GAG production (Fig. 3i). In addition, pretreatment with U0126 or SB203580 also suppressed the shockwave-induced gene expression of Col2a1 and Acan (Fig. 4a-b). However, shockwaves had no influence on the gene expression of Col10a1 with or without these inhibitors. Elevated protein expression of Col2a1 and Acan, as well as GAG synthesis, returned to basal levels after the inhibition of Erk signaling (Fig. 4c-d). Taken together, these results indicated that shockwave-induced transient ROS production was mainly from XO and was crucial for the subsequent signal transduction of MAPK and increased the production of ECM in porcine chondrocytes.

\section{Shockwaves activated the redox activity of Nrf2 in porcine chondrocytes}

Nrf2, the master regulator of redox activity, regulates the expression of a large and complex network of cytoprotective genes[19]. Because the shockwaves gave rise to the transient low-level ROS signal, we subsequently examined the adaptive response of redox activity in chondrocytes after shockwave treatment. Western blotting revealed no significant elevation of total Nrf2 protein within $72 \mathrm{~h}$ after shockwave treatment (Fig. 5a). However, a significant accumulation of Nrf2 in the nucleus was observed at $16 \mathrm{~h}$ and was sustained for $20 \mathrm{~h}$ after shockwave treatment by using immunoblotting and immunofluorescence methods (Fig. 5b-c). Furthermore, Nqo-1 and Ho-1 are Nrf2-dependent antioxidant genes involved in preventing oxidative stress. Our results show that the downstream gene expression of 
Nrf2 effectors, including Ho-1 and Nqo-1, were elevated in a dose- and time-dependent manner in shockwave-treated porcine chondrocyte pellets (Fig. 5d-f). The time-series study revealed that the gene expression of Ho-1 and Nqo-1 were gradually elevated and peaked at $18 \mathrm{~h}$ after shockwave treatment.

\section{Shockwave-induced ROS and MAPK signaling activated the nuclear translocation of Nrf2 and the Nrf2- dependent antioxidant and chondrogenic gene expression}

The shockwave-induced transient ROS signaling plays an important role in the redox activity of Nrf2 in porcine chondrocytes. The immunofluorescence study revealed that porcine chondrocytes pretreated with allopurinol reduced the shockwave-activated nuclear translocation of Nrf2, and the gene expression of Ho-1 and Nqo-1 also returned to the basal level (Fig. 6a-b).

Previous studies have shown that Nrf2 nuclear translocation activation involves the MAPK signaling pathway[21]. Our immunofluorescence study revealed that U0126 and SB203580 reduced the shockwaveinduced nuclear translocation of Nrf2 (Fig. 6c-d). The shockwave-elevated gene expression of Ho-1 and Nqo-1 returned to the basal level after MAPK signaling was blocked with either U0126 or SB203580 (Fig. 6e-f).

To further substantiate the role of Nrf2 in shockwave-induced ECM synthesis, we depleted Nrf2 activity using brusatol and the knockdown of Nrf2 using Nrf2-specific shNrf2-expressing lentiviral plasmid. Brusatol extracted from Brucea javanica has been shown to rapidly and transiently inhibit the Nrf2mediated signaling pathway independently, without altering the activity of Keap1[31].The shockwaveinduced protein expression of Col2a1 and Acan, as well as GAG production, was abrogated by pretreatment of porcine chondrocytes with brusatol (Fig. 6g-h). Furthermore, porcine chondrocytes with knockdown of Nrf2 also had no significantly elevated protein production of Col2a1 and Acan after shockwave treatment (Fig. 6i).

Altogether, these data indicate that shockwaves might increase Nrf2 activity through the activation of ROS and MAPK signaling. The shockwave-elevated redox activity of Nrf2 had direct effects on the shockwave-induced enhancement of ECM production in porcine chondrocytes.

\section{Shockwaves increased GAG production of human OA chondrocytes}

To further examine whether the beneficial effect of shockwave treatment in porcine articular chondrocytes can be applied for clinical use, we further use the human OA chondrocytes to confirm the therapeutic effect of shockwaves for OA. The human OA chondrocytes were also cultured in pellets and subjected to shockwave treatment as described above for the porcine experiments. Similar results were also observed in human OA chondrocyte samples. After three shockwave treatments within $1 \mathrm{wk}$, the human OA chondrocyte pellets still permitted $>90 \%$ cell viability as detected by alamarBlue assay at different time points after shockwave treatment (Fig. 7a). There was also no significant difference in the 
total DNA amount of human OA chondrocyte pellets between the control and any shockwave treatment group (Fig. 7b).

The GAG synthesis of human OA chondrocyte pellets was significantly elevated after shockwave treatment compared with the untreated control group (Fig. 7c). Shockwave treatment had a doseresponse relationship with GAG production. The increases in GAG production were more prominent after five shockwave treatments within the same duration compared with the samples subjected to three shockwave treatments. Taken together, these results suggest that shockwave treatment can enhance the ECM production of both porcine and human OA chondrocytes without affecting cell viability or proliferation.

\section{Discussion}

Multiple studies have reported that shockwave treatment has therapeutic potential for the management of $O A[1,5,25]$. However, the biological response and mechanism of shockwave treatment on chondrocyte remain unclear, which makes it difficult to develop an efficient treatment for OA. In this study, we used the 3D cell culture model to investigate the effect of shockwaves on the ECM synthesis of chondrocytes and to further define the signal transduction pathway. The most significant finding of the present study is that shockwave treatment could significantly enhance the ECM production of chondrocytes, and this beneficial effect was associated with the ROS/MAPK/Nrf2 signaling pathway (Fig. 8). Identification of the shockwave-induced biological effect and related mechanisms can facilitate further investigations aimed at developing novel treatment strategies.

Previous studies have indicated that shockwaves might increase the cytotoxicity of articular chondrocytes in an energy- and impulse-dependent manner; an energy flux density (EFD) higher than $0.06 \mathrm{~mJ} / \mathrm{mm}^{2}$ or excessive impulses of shockwaves might endanger chondrocyte viability [1, 32, 33]. These results were obtained from 2D chondrocyte culture subjected to shockwave treatment. However, Renz et al. embedded human chondrocytes in alginate beads and found no significant elevation of cytotoxicity with $0.26 \mathrm{~mJ} / \mathrm{mm}^{2}$, whereas cell death was significantly increased when chondrocytes were prepared in a fluid suspension[34]. Our results also confirmed the safety of applying shockwaves to chondrocytes at certain energy levels (500 impulses at $0.3 \mathrm{~mJ} / \mathrm{mm}^{2}$ ). In previous animal studies, few gross pathologic changes were observed in the joints after shockwave treatment with EFD and impulses within commonly used ranges for another musculoskeletal disease ${ }^{[35,36]}$. Moreover, previous clinic trials have also indicated that most patients could tolerate up to 4000 impulses below $0.4 \mathrm{~mJ} / \mathrm{mm}^{2}[3,4,25]$. Collectively, these data support the safety of the clinical use of shockwave treatment for degenerative cartilage disease.

Previous studies revealed a significant downregulation of proteoglycan synthesis gene expression at the onset of $\mathrm{OA}$ in a rat model, and this finding was also consistent with the observation in human $O A$ samples with normal appearance[37,38]. The reduced proteoglycan content not only predisposed the cartilage to greater strains when exposed to mechanical stress but also induced a series of downstream 
activities, including ECM remodeling and MMP13 induction after discoidin domain receptors were uncovered[38-40]. In the present study, shockwaves enhanced the GAG synthesis of human OA chondrocytes without significantly affecting cell viability and proliferation. These results were in agreement with those of previous animal studies demonstrating that shockwave treatment improved safranin-O staining in the proximal medial tibia of OA knees induced by ACLT and osteoporotic OA induced by bilateral ovariectomies in rats $[7,9,41]$. Wang et al. proposed that shockwave treatment might have a chondroprotective effect, and this beneficial effect was associated with subchondral bone turnover[6, 7, 9, 42]. In the present study, we demonstrated that the chondrocytes could independently sense the physical signal of shockwaves and translate it into a biological response.

Previous studies have indicated that ROS inhibited chondrocyte proliferation and modulated the initiation of the hypertrophic changes in chondrocytes[43]. Furthermore, the ROS-activated MAPK pathway suppressed the expression of Col2a1 and Acan in chondrocytes[44, 45]. Our data revealed that ROS/MAPK kinase/Nrf2 signaling is crucial for the shockwave-induced ECM synthesis of chondrocytes. A possible explanation for this is that ROS play a dual role in the regulation of cartilage homeostasis; low physiological levels of ROS might serve as important cellular messengers that regulate physiological processes[14-18, 46]. The balance between ROS and intracellular antioxidant levels is pivotal for maintaining the homeostasis of cartilage tissue. Moreover, the ROS signaling induced by shockwaves was transient and intermittent, which is different from the pharmacologic induction of oxidative stress in experimental settings $[44,47]$. Identification of the optimal level and stimulation interval of ROS signaling is important for further clinical application.

The early detection of ROS elevation (usually within $30 \mathrm{~min}$ ) is consistent with the previous finding that ROS is a crucial upstream signal for initiating the shockwave-induced biological response [12-14]. Wang et al. found that NADPH oxidase was the main source of shockwave-induced ROS production in osteoblasts, but our results demonstrated that XO was mainly responsible for ROS production in chondrocytes[14]. An increase in XO activity was observed in the synovial membrane of individuals with acute joint injury and metabolic arthritis, but little is known about the role of $\mathrm{XO}$ activation in chondrocytes[28, 48,49]. XO generates $\mathrm{H}_{2} \mathrm{O}_{2}$, which is freely diffusible and important for intra- and intercellular signal transduction[28,50]. Further studies must be undertaken to identify whether $\mathrm{H}_{2} \mathrm{O}_{2}$ is the key mediator that transfers the mechanical signal of shockwaves from the focal working zone to the surrounding area through the autocrine and paracrine effects in chondrocytes. ROS induced by lowconcentration $\mathrm{XO}$ or another exogenous oxidant frequently stimulated the proteoglycan synthesis of articular chondrocytes, but a higher concentration inhibited these effects $[46,51,52]$.

Although the mechanism by which ROS can trigger the MAPK pathway was not well defined, previous studies have indicated that the various cellular stimuli that elevate ROS production could trigger activation of the MAPK pathway[29, 53,54]. Our finding that the phosphorylation of MAPK was responsible for shockwave-induced ROS signaling in various cell types is consistent with findings in the literature[12-14, 47]. We found that the shockwaves activated the phosphorylation of Erk and p38 MAPK in chondrocytes through the production of ROS. Interestingly, others have reported that shockwaves 
activated Erk and p38 signaling through cellular ATP release and P2 receptor stimulation in osteoblasts, mesenchymal stem cells, and T cells[30,55,56]. The connection between shockwave-induced ROS production and cellular ATP release in signaling cascades requires further investigation.

Nrf2 is the master regulator of antioxidant responses and is under consideration as a therapeutic target for $\mathrm{OA}[19-21]$. Both overexpression and downregulation of Nrf2 can lead to abnormal chondrogenesis; an appropriate Nrf2 level is necessary for cartilage homeostasis[57, 58]. Recently, studies have demonstrated that the pharmacological activation or genetic overexpression of Nrf2 and Nrf2-dependent genes could reduce IL-1 $\beta$-stimulated oxidative stress, inflammation, and matrix degradation[20, 21, 59]. Several transcription factors have been found to regulate Col2a1 through its promoter, including Sp1, Smad3/4, AP1, and nuclear factor-kB. Our search of the TRANSFAC database (geneXplain, Wolfenbüttel, Germany) revealed that the Nrf2 consensus core sequence (5'-nTGAnTCAGC $n-3$ ', where $n=\mathrm{A}, \mathrm{C}, \mathrm{G}$ or T) is present in the Col2a1 promoter, suggesting that Nrf2 might regulate Col2a1 expression through directly binding to the consensus sequence or through indirect cross-talk with other transcriptional factors. Elucidation of the actual mechanism requires further exploration. In the present study, we reported that the nonpharmacologic activation of Nrf2, Ho-1, and Nqo-1 by shockwaves could increase the ECM synthesis of articular chondrocytes without pretreatment with any cytokines. However, recent studies have also revealed that the expression of Nrf2 and its Nrf2-dependent genes, such as Ho-1 and Nqo-1, was significantly higher in $\mathrm{OA}$ joints and the damage zone of human cartilage than in normal cartilage[20]. Moreover, the Nrf2 and Ho-1 expression of OA cartilage from patients with type 2 diabetes mellitus (T2DM) were reduced compared with the sample from non-T2DM patients[60]. The impairment of Nrf2 related antioxidant system may lead to greater inflammatory reaction of OA cartilage from T2DM patients. In a further clinical trial on shockwave treatment for $\mathrm{OA}$, the optimal candidate should also be carefully selected for investigation.

\section{Conclusions}

Although recent studies have indicated that shockwave treatment could benefit patients with $O A$, the molecular mechanisms underlying these beneficial effects have remained unclear. We believe that our findings provide subjective evidence to support the clinical use of shockwave treatment for OA. Our results suggest that shockwaves increase ECM production in both healthy porcine articular chondrocytes and human OA chondrocytes without affecting the cell viability and proliferation. As summarized in Fig. 8, we propose that shockwave-induced anabolic response occurs through the induction of ROS and activation of Nrf2/ARE pathways in articular chondrocytes. The shockwave-induced transient ROS signal was mainly from XO and subsequently induced the nuclear translocation of $\mathrm{Nrf2}$, the downstream gene expression of Ho-1 and Nqo-1, and ECM synthesis. New insight into the mechanism of chondrocyte react to the shockwave stimulation will facilitate further strategy development of shockwave treatment for OA.

\section{Abbreviations}


Acan, Aggrecan; ACLT, Anterior cruciate ligament transection; AREs, Antioxidant response elements; BSA, Bovine serum albumin; Col10a1, Collagen, type $X$, a1 chain; Col2a1, Collagen, type II, a1 chain; $C_{t}$, Threshold cycle; DCF, Dichlorofluorescein; DCFH-DA, Dichlorofluorescein diacetate; DMEM, Dulbecco's modified Eagle's medium; DPI, Diphenyleneiodonium; DTT, Dithiothreitol; ECM, Extracellular matrix; EDTA, Ethylenediaminetetraacetic acid; EFD, Energy flux density; Erk, Extracellular signal-regulated kinase; FCS, Fetal calf serum; GAG, sulfated glycosaminoglycan; GAPDH, Glyceraldehyde 3-phosphate dehydrogenase; HEPES, 4-(2-Hydroxyethyl)-1-piperazineethanesulfonic acid; Ho-1, Heme oxygenase-1; lgG, Immunoglobulin G; IL-10, Interleukin-10; MAPK, Mitogen-activated protein kinase; MMP13, Matrix metallopeptidase 13; NADPH, Nicotinamide adenine dinucleotide phosphate; Nqo-1, NAD(P)H:quinone oxidoreductase; Nrf2, Nuclear factor erythroid 2-related factor; OA, Osteoarthritis; PBS, Phosphatebuffered saline; PCM, Pericellular matrix; PCR, Polymerase chain reaction; ROS, Reactive oxygen species; SDS-PAGE, Sodium dodecyl sulfate-polyacrylamide gel electrophoresis; shRNA, Small hairpin RNA; siRNA, Small interfering RNA; T2DM, Type 2 diabetes mellitus; XO, Xanthine oxidase

\section{Declarations}

\section{Ethical Approval and Consent to participate}

This study was approved by the Institutional Review Board of Kaohsiung Medical University Chung-Ho Memorial Hospital (KMUH-IRB-20140144).

\section{Consent for publication}

Not applicable.

\section{Availability of Data and Materials}

The datasets used and/or analyzed during the current study are available from the corresponding author on reasonable request.

\section{Competing interests}

The authors declare that they have no competing interests.

\section{Funding}

Not applicable.

\section{Authors contribution}

All authors were involved in drafting the article critically for important intellectual content. PCS, and YCT had full access to all of the data in the study and take responsibility for the integrity of the data and the accuracy of the data analysis. Study conception and design. HTH, ZML, CCL, CLS, LMC. Acquisition of 
data. SH, Chien; SH, Chou. Analysis and interpretation of data. PCS, PJH, SJS, YCT. All authors read and approved the final manuscript.

\section{Acknowledgments}

This work was supported by research grant from the Ministry of Science and Technology (104-2314-B037-038-MY3) and from Kaohsiung Medical University Hospital (KMUH104-4R41). This manuscript was edited by Wallace Academic Editing.

\section{Authors' information}

1. Department of Orthopaedic Surgery, Kaohsiung Medical University Hospital, Kaohsiung, Taiwan. 2. Graduate Institute of Medicine, College of Medicine, Kaohsiung Medical University, Kaohsiung, Taiwan. 3. Department of Orthopaedic Surgery, Kaohsiung Municipal Siaoqang Hospital, Kaohsiung, Taiwan. 4. Department of Orthopaedic Surgery, Kaohsiung Municipal Ta-Tung Hospital, Kaohsiung, Taiwan. 5. Department of Medical Laboratory Science and Biotechnology, School of Medicine and Health Sciences, Fooyin University, Kaohsiung, Taiwan. 6. Departments of Pediatrics, E-DA Hospital, IShou University, Kaohsiung City, Taiwan

\section{References}

1. Ji Q, Wang P, He C. Extracorporeal shockwave therapy as a novel and potential treatment for degenerative cartilage and bone disease: Osteoarthritis. A qualitative analysis of the literature. Prog Biophys Mol Biol. 2016;121:255-65.

2. Ji Q, He C. Extracorporeal shockwave therapy promotes chondrogenesis in cartilage tissue engineering: A hypothesis based on previous evidence. Med Hypotheses. 2016;91:9-15.

3. Zhao Z, Jing R, Shi Z, Zhao B, Ai Q, Xing G. Efficacy of extracorporeal shockwave therapy for knee osteoarthritis: a randomized controlled trial. J Surg Res. 2013;185:661-6.

4. Chen TW, Lin CW, Lee CL, Chen CH, Chen YJ, Lin TY, Huang MH. The efficacy of shock wave therapy in patients with knee osteoarthritis and popliteal cyamella. Kaohsiung J Med Sci. 2014;30:362-70.

5. Wang YC, Huang HT, Huang PJ, Liu ZM, Shih CL. Efficacy and Safety of Extracorporeal Shockwave Therapy for Treatment of Knee Osteoarthritis: A Systematic Review and Meta-analysis. Pain Med 2019.

6. Wang CJ, Weng LH, Ko JY, Sun YC, Yang YJ, Wang FS. Extracorporeal shockwave therapy shows chondroprotective effects in osteoarthritic rat knee. Arch Orthop Trauma Surg. 2011;131:1153-8.

7. Wang CJ, Weng LH, Ko JY, Wang JW, Chen JM, Sun YC, Yang YJ. Extracorporeal shockwave shows regression of osteoarthritis of the knee in rats. J Surg Res. 2011;171:601-8.

8. Zhao Z, Ji H, Jing R, Liu C, Wang M, Zhai L, Bai X, Xing G. Extracorporeal shock-wave therapy reduces progression of knee osteoarthritis in rabbits by reducing nitric oxide level and chondrocyte apoptosis. Arch Orthop Trauma Surg. 2012;132:1547-53. 
9. Wang CJ, Sun YC, Wong T, Hsu SL, Chou WY, Chang HW. Extracorporeal shockwave therapy shows time-dependent chondroprotective effects in osteoarthritis of the knee in rats. J Surg Res. 2012;178:196-205.

10. Moretti B, lannone F, Notarnicola A, Lapadula G, Moretti L, Patella V, Garofalo R. Extracorporeal shock waves down-regulate the expression of interleukin-10 and tumor necrosis factor-alpha in osteoarthritic chondrocytes. BMC Musculoskelet Disord. 2008;9:16.

11. Ma B, Leijten JC, Wu L, Kip M, van Blitterswijk CA, Post JN, Karperien M. Gene expression profiling of dedifferentiated human articular chondrocytes in monolayer culture. Osteoarthritis Cartilage. 2013;21:599-603.

12. Catalano MG, Marano F, Rinella L, de Girolamo L, Bosco O, Fortunati N, Berta L, Frairia R. Extracorporeal shockwaves (ESWs) enhance the osteogenic medium-induced differentiation of adipose-derived stem cells into osteoblast-like cells. J Tissue Eng Regen Med. 2017;11:390-9.

13. Wang FS, Wang CJ, Sheen-Chen SM, Kuo YR, Chen RF, Yang KD. Superoxide mediates shock wave induction of ERK-dependent osteogenic transcription factor (CBFA1) and mesenchymal cell differentiation toward osteoprogenitors. J Biol Chem. 2002;277:10931-7.

14. Wang FS, Wang CJ, Chen YJ, Chang PR, Huang YT, Sun YC, Huang HC, Yang YJ, Yang KD. Ras induction of superoxide activates ERK-dependent angiogenic transcription factor HIF-1alpha and VEGF-A expression in shock wave-stimulated osteoblasts. J Biol Chem. 2004;279:10331-7.

15. Lepetsos P, Papavassiliou AG. ROS/oxidative stress signaling in osteoarthritis. Biochim Biophys Acta. 2016;1862:576-91.

16. Henrotin Y, Kurz B, Aigner T. Oxygen and reactive oxygen species in cartilage degradation: friends or foes? Osteoarthritis Cartilage. 2005;13:643-54.

17. Henrotin Y, Deby-Dupont G, Deby C, De Bruyn M, Lamy M, Franchimont P. Production of active oxygen species by isolated human chondrocytes. Br J Rheumatol. 1993;32:562-7.

18. Li J, Dong S. The Signaling Pathways Involved in Chondrocyte Differentiation and Hypertrophic Differentiation. Stem Cells Int. 2016;2016:2470351.

19. Marchev AS, Dimitrova PA, Burns AJ, Kostov RV, Dinkova-Kostova AT, Georgiev Ml. Oxidative stress and chronic inflammation in osteoarthritis: can NRF2 counteract these partners in crime? Ann N Y Acad Sci. 2017;1401:114-35.

20. Khan NM, Ahmad I, Haqqi TM. Nrf2/ARE pathway attenuates oxidative and apoptotic response in human osteoarthritis chondrocytes by activating ERK1/2/ELK1-P70S6K-P90RSK signaling axis. Free Radic Biol Med. 2018;116:159-71.

21. Khan NM, Haseeb A, Ansari MY, Devarapalli P, Haynie S, Haqqi TM. Wogonin, a plant derived small molecule, exerts potent anti-inflammatory and chondroprotective effects through the activation of ROS/ERK/Nrf2 signaling pathways in human Osteoarthritis chondrocytes. Free Radic Biol Med. 2017;106:288-301.

22. Cai D, Yin S, Yang J, Jiang Q, Cao W. Histone deacetylase inhibition activates Nrf2 and protects against osteoarthritis. Arthritis Res Ther. 2015;17:269. 
23. Khan NM, Haqqi TM. Pleiotropic Roles of Nrf2 as Regulators of Chondrocyte Apoptosis, Oxidative Stress, Inflammatory Response and Catabolic and Anabolic Pathways in Osteoarthritis. Free Radic Biol Med. 2017;112:191-1.

24. Sun YX, Li L, Corry KA, Zhang P, Yang Y, Himes E, Mihuti CL, Nelson C, Dai G, Li J. Deletion of Nrf2 reduces skeletal mechanical properties and decreases load-driven bone formation. Bone. 2015;74:19.

25. Li W, Pan Y, Yang Q, Guo ZG, Yue Q, Meng QG. Extracorporeal shockwave therapy for the treatment of knee osteoarthritis: A retrospective study. Medicine. 2018;97:e11418.

26. Wu L, Leijten JC, Georgi N, Post JN, van Blitterswijk CA, Karperien M. Trophic effects of mesenchymal stem cells increase chondrocyte proliferation and matrix formation. Tissue Eng Part A. 2011;17:1425-36.

27. Liu L, Zhang K, Sandoval H, Yamamoto S, Jaiswal M, Sanz E, Li Z, Hui J, Graham BH, Quintana A, Bellen HJ. Glial lipid droplets and ROS induced by mitochondrial defects promote neurodegeneration. Cell. 2015;160:177-90.

28. Bolduc JA, Collins JA, Loeser RF. Reactive oxygen species, aging and articular cartilage homeostasis. Free Radic Biol Med. 2019;132:73-82.

29. Son Y, Cheong YK, Kim NH, Chung HT, Kang DG, Pae HO. Mitogen-Activated Protein Kinases and Reactive Oxygen Species: How Can ROS Activate MAPK Pathways? J Signal Transduct. 2011;2011:792639.

30. Weihs AM, Fuchs C, Teuschl AH, Hartinger J, Slezak P, Mittermayr R, Redl H, Junger WG, Sitte HH, Runzler D. Shock wave treatment enhances cell proliferation and improves wound healing by ATP release-coupled extracellular signal-regulated kinase (ERK) activation. J Biol Chem. 2014;289:27090-104.

31. Vartanian S, Ma TP, Lee J, Haverty PM, Kirkpatrick DS, Yu K, Stokoe D. Application of Mass Spectrometry Profiling to Establish Brusatol as an Inhibitor of Global Protein Synthesis. Mol Cell Proteomics. 2016;15:1220-31.

32. Dorotka R, Kubista B, Schatz KD, Trieb K. Effects of extracorporeal shock waves on human articular chondrocytes and ovine bone marrow stromal cells in vitro. Arch Orthop Trauma Surg. 2003;123:345-8.

33. Byron CR, Benson BM, Stewart AA, Stewart MC. Effects of radial shock waves on membrane permeability and viability of chondrocytes and structure of articular cartilage in equine cartilage explants. Am J Vet Res. 2005;66:1757-63.

34. Renz H, Rupp S. Effects of shock waves on chondrocytes and their relevance in clinical practice. Arch Orthop Trauma Surg. 2009;129:641-7.

35. Vaterlein N, Lussenhop S, Hahn M, Delling G, Meiss AL. The effect of extracorporeal shock waves on joint cartilage-an in vivo study in rabbits. Arch Orthop Trauma Surg. 2000;120:403-6.

36. Mayer-Wagner S, Ernst J, Maier M, Chiquet M, Joos H, Muller PE, Jansson V, Sievers B, Hausdorf J. The effect of high-energy extracorporeal shock waves on hyaline cartilage of adult rats in vivo. $J$ 
Orthop Res. 2010;28:1050-6.

37. Nam J, Perera P, Liu J, Rath B, Deschner J, Gassner R, Butterfield TA, Agarwal S. Sequential alterations in catabolic and anabolic gene expression parallel pathological changes during progression of monoiodoacetate-induced arthritis. PLoS One. 2011;6:e24320.

38. Maldonado $\mathrm{M}, \mathrm{Nam} \mathrm{J}$. The role of changes in extracellular matrix of cartilage in the presence of inflammation on the pathology of osteoarthritis. Biomed Res Int. 2013;2013:284873.

39. Madry H, Luyten FP, Facchini A. Biological aspects of early osteoarthritis. Knee Surg Sports Traumatol Arthrosc. 2012;20:407-22.

40. Vogel WF, Abdulhussein R, Ford CE. Sensing extracellular matrix: an update on discoidin domain receptor function. Cell Signal. 2006;18:1108-16.

41. Wang CJ, Huang CY, Hsu SL, Chen JH, Cheng JH. Extracorporeal shockwave therapy in osteoporotic osteoarthritis of the knee in rats: an experiment in animals. Arthritis Res Ther. 2014;16:R139.

42. Wang CJ, Sun YC, Siu KK, Wu CT. Extracorporeal shockwave therapy shows site-specific effects in osteoarthritis of the knee in rats. J Surg Res. 2013;183:612-9.

43. Morita K, Miyamoto T, Fujita N, Kubota Y, Ito K, Takubo K, Miyamoto K, Ninomiya K, Suzuki T, Iwasaki $\mathrm{R}$, et al. Reactive oxygen species induce chondrocyte hypertrophy in endochondral ossification. J Exp Med. 2007;204:1613-23.

44. Yu SM, Kim SJ. Production of reactive oxygen species by withaferin A causes loss of type collagen expression and COX-2 expression through the PI3K/Akt, p38, and JNK pathways in rabbit articular chondrocytes. Exp Cell Res. 2013;319:2822-34.

45. Goodwin W, McCabe D, Sauter E, Reese E, Walter M, Buckwalter JA, Martin JA. Rotenone prevents impact-induced chondrocyte death. J Orthop Res. 2010;28:1057-63.

46. Lee RB, Urban JP. Functional replacement of oxygen by other oxidants in articular cartilage. Arthritis Rheum. 2002;46:3190-200.

47. Collins JA, Wood ST, Nelson KJ, Rowe MA, Carlson CS, Chubinskaya S, Poole LB, Furdui CM, Loeser RF. Oxidative Stress Promotes Peroxiredoxin Hyperoxidation and Attenuates Pro-survival Signaling in Aging Chondrocytes. J Biol Chem. 2016;291:6641-54.

48. Stabler T, Zura RD, Hsueh MF, Kraus VB. Xanthine oxidase injurious response in acute joint injury. Clin Chim Acta. 2015;451:170-4.

49. Aibibula Z, Ailixiding M, Iwata M, Piao J, Hara Y, Okawa A, Asou Y. Xanthine oxidoreductase activation is implicated in the onset of metabolic arthritis. Biochem Biophys Res Commun. 2016;472:26-32.

50. Klomsiri C, Karplus PA, Poole LB. Cysteine-based redox switches in enzymes. Antioxid Redox Signal. 2011;14:1065-77.

51. Panasyuk A, Frati E, Ribault D, Mitrovic D. Effect of reactive oxygen species on the biosynthesis and structure of newly synthesized proteoglycans. Free Radic Biol Med. 1994;16:157-67. 
52. Ramakrishnan P, Hecht BA, Pedersen DR, Lavery MR, Maynard J, Buckwalter JA, Martin JA. Oxidant conditioning protects cartilage from mechanically induced damage. J Orthop Res. 2010;28:914-20.

53. Torres M, Forman HJ. Redox signaling and the MAP kinase pathways. Biofactors. 2003;17:287-96.

54. McCubrey JA, Lahair MM, Franklin RA. Reactive oxygen species-induced activation of the MAP kinase signaling pathways. Antioxid Redox Signal. 2006;8:1775-89.

55. Sun D, Junger WG, Yuan C, Zhang W, Bao Y, Qin D, Wang C, Tan L, Qi B, Zhu D, et al. Shockwaves induce osteogenic differentiation of human mesenchymal stem cells through ATP release and activation of P2 $\times 7$ receptors. Stem Cells. 2013;31:1170-80.

56. Yu T, Junger WG, Yuan C, Jin A, Zhao Y, Zheng X, Zeng Y, Liu J. Shockwaves increase T-cell proliferation and IL-2 expression through ATP release, $\mathrm{P} 2 \times 7$ receptors, and FAK activation. Am $\mathrm{J}$ Physiol Cell Physiol. 2010;298:C457-64.

57. Hinoi E, Takarada T, Fujimori S, Wang L, lemata M, Uno K, Yoneda Y. Nuclear factor E2 p45-related factor 2 negatively regulates chondrogenesis. Bone. 2007;40:337-44.

58. Solomon LA, Berube NG, Beier F. Transcriptional regulators of chondrocyte hypertrophy. Birth Defects Res C Embryo Today. 2008;84:123-30.

59. Park C, Hong SH, Shin SS, Lee DS, Han MH, Cha HJ, Kim S, Kim HS, Kim GY, Park EK, et al: Activation of the Nrf2/HO-1 Signaling Pathway Contributes to the Protective Effects of Sargassum serratifolium Extract against Oxidative Stress-Induced DNA Damage and Apoptosis in SW1353 Human Chondrocytes. Int J Environ Res Public Health 2018, 15.

60. Vaamonde-Garcia C, Courties A, Pigenet A, Laiguillon MC, Sautet A, Houard X, Kerdine-Romer S, Meijide R, Berenbaum F, Sellam J. The nuclear factor-erythroid 2-related factor/heme oxygenase-1 axis is critical for the inflammatory features of type 2 diabetes-associated osteoarthritis. J Biol Chem. 2017;292:14505-15.

\section{Figures}


a

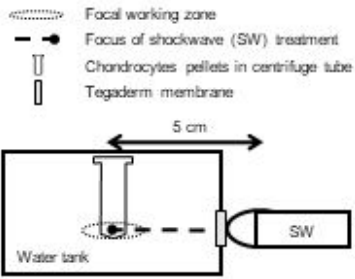

d

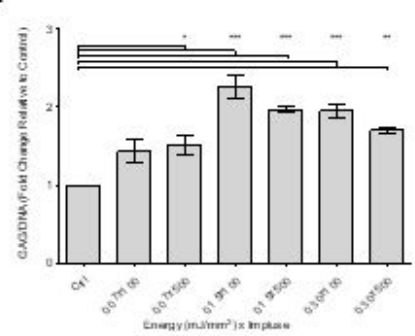

g

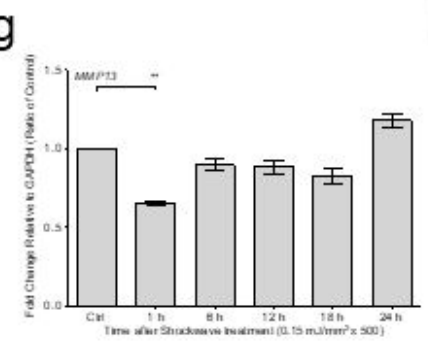

b

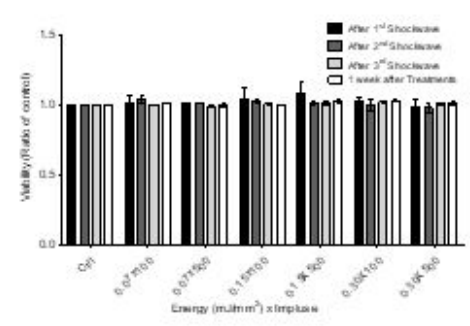

e
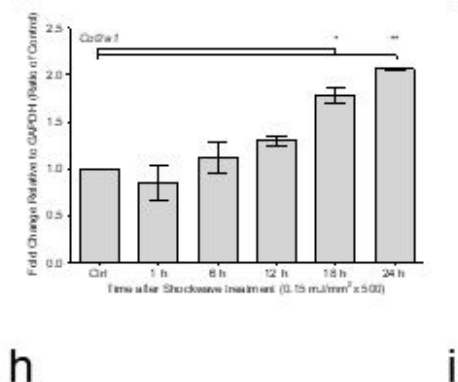

C

f

i
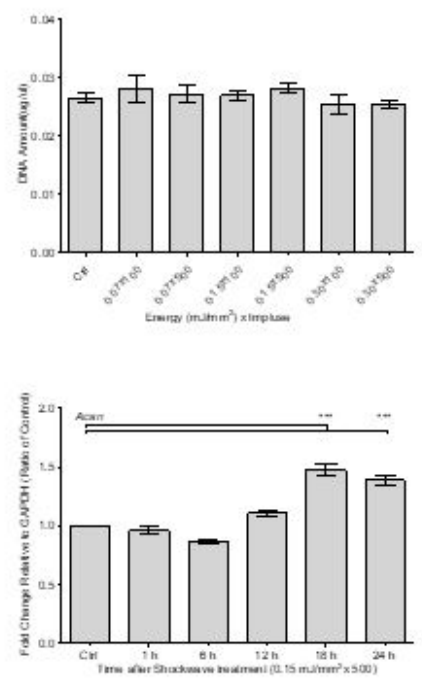

Shockwave treatment
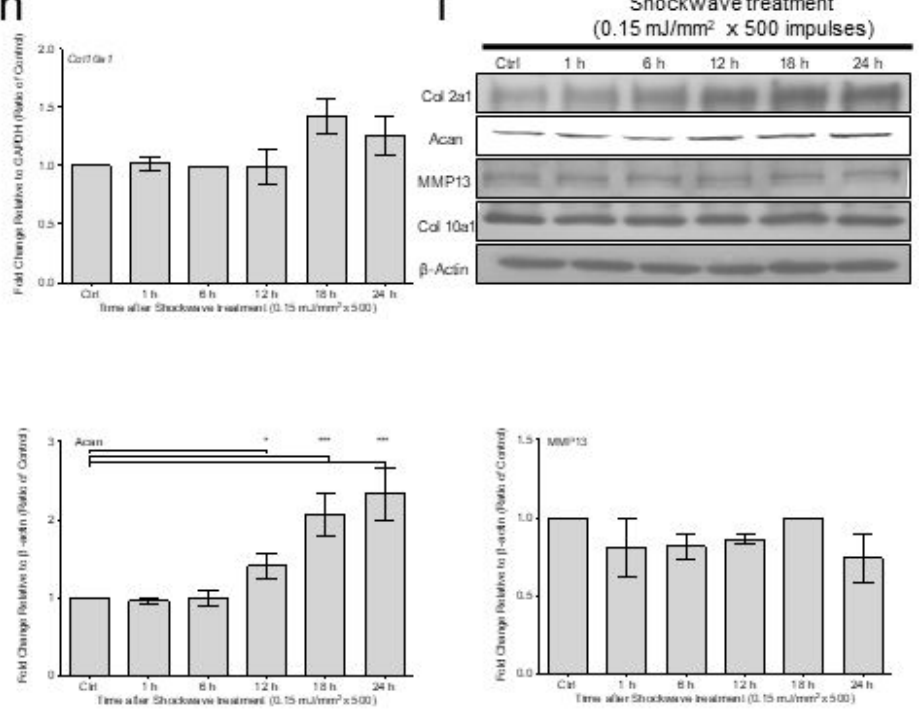

j

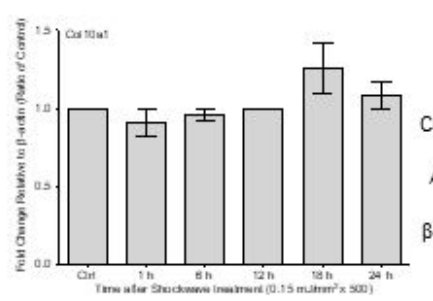

Shockwave treatment
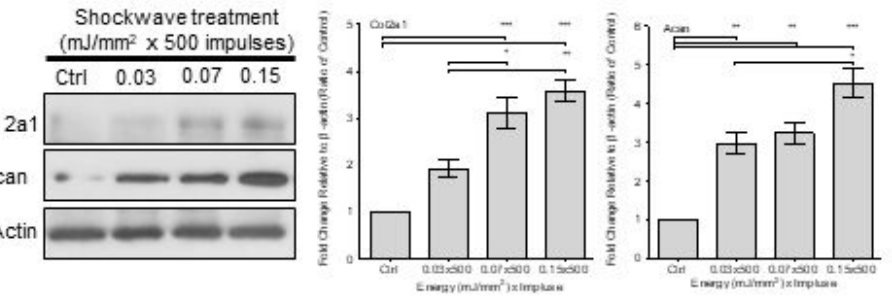

\section{Figure 1}

Shockwaves enhance the extracellular matrix synthesis of porcine chondrocytes without affecting cell viability. To assess the biological function of chondrocytes after shockwave treatment, the porcine chondrocytes were cultured in pellets for $5 \mathrm{~d}$ before shockwave treatment. The samples were subjected to three focused shockwave treatments using model A with the indicated dosage within 1 wk and then continued to be cultured for an additional $7 \mathrm{~d}$. a A schematic of the model A setup. b The cell viability at 
different time points was investigated by alamarBlue assay. $c$ The total DNA content of cell pellets was measured by Hoechst DNA assay at $7 \mathrm{~d}$ after the latest treatment. $\mathrm{d}$ To determine the dose-dependent activation of total sulfated glycosaminoglycan (GAG) synthesis after shockwave treatment, the cell pellets and all the culture supernatants were collected for measurement of total sulfated GAG content using the 1,9-dimethylmethylene blue method. The values of GAG were normalized by the DNA content of each sample. e-h Gene expression of Col2a1, Acan, MMP13, and Col10a1 after single shockwave treatment (500 impulses at $0.15 \mathrm{~mJ} / \mathrm{mm} 2$ ) at the indicated time points was measured by real-time polymerase chain reaction. i Time-course comparison of protein expression of Col2a1, Acan, MMP13, and Col10a1 after single shockwave treatment (500 impulses at $0.15 \mathrm{~mJ} / \mathrm{mm} 2$ ). j Dose-dependent protein expression of Col2a1 and Acan at $24 \mathrm{~h}$ after shockwave treatment with the indicated dosage. (Each bar represents mean \pm SEM, *: $p<.05 ; * \star: p<.01 ; * \star *: p<.001 ; S W$ : shockwave treatment) 

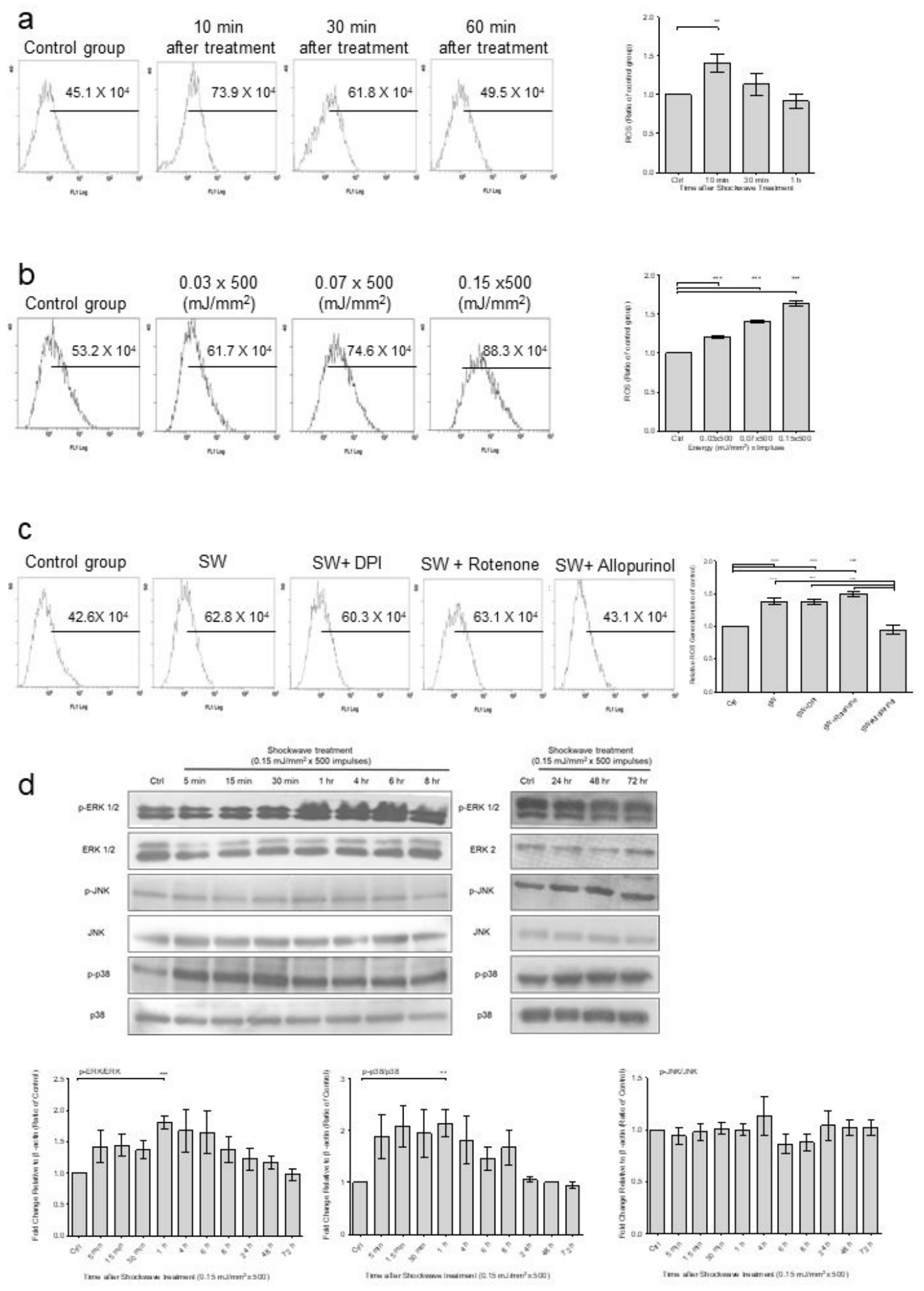

\section{Figure 2}

Shockwave-induced reactive oxygen species (ROS) production of porcine chondrocytes in a time- and dose-dependent fashion. Porcine chondrocyte cell pellets were treated with a single course of shockwave treatment. For the detection of ROS, the porcine chondrocyte pellets were trypsinized and washed with ice-cold phosphate-buffered saline to quantify the fluorescence emitted by fluorescence-activated cell sorting. a Time course of ROS production after shockwave treatment with 500 impulses at 0.15 
$\mathrm{mJ} / \mathrm{mm} 2$. b ROS level at $10 \mathrm{~min}$ after shockwave treatment with different dosages. c To determine the main source of shockwave-induced ROS production, rotenone (inhibits mitochondrial complex I), the porcine chondrocytes were pretreated with diphenyleneiodonium (inhibits nicotinamide adenine dinucleotide phosphate oxidase) or allopurinol (inhibits xanthine oxidase) before the single shockwave treatment (500 impulses at $0.15 \mathrm{~mJ} / \mathrm{mm} 2$ ). $\mathrm{d}$ Western blotting was performed to investigate the phosphorylation of mitogen-activated protein kinases (MAPKs; Erk, p38, and JNK) at the indicated time points induced by single shockwave treatment (500 impulses at $0.15 \mathrm{~mJ} / \mathrm{mm} 2$ ). The ratio of phosphorylated MAPK to total MAPK was calculated by densitometry in each sample, and a value of 1 was given to the control samples. (Each bar represents mean \pm SEM, $*$ : $<.05 ; * \star: p<.01 ; * \star *: p<.001$; Ctrl: Control group; SW: shockwave treatment) 
a

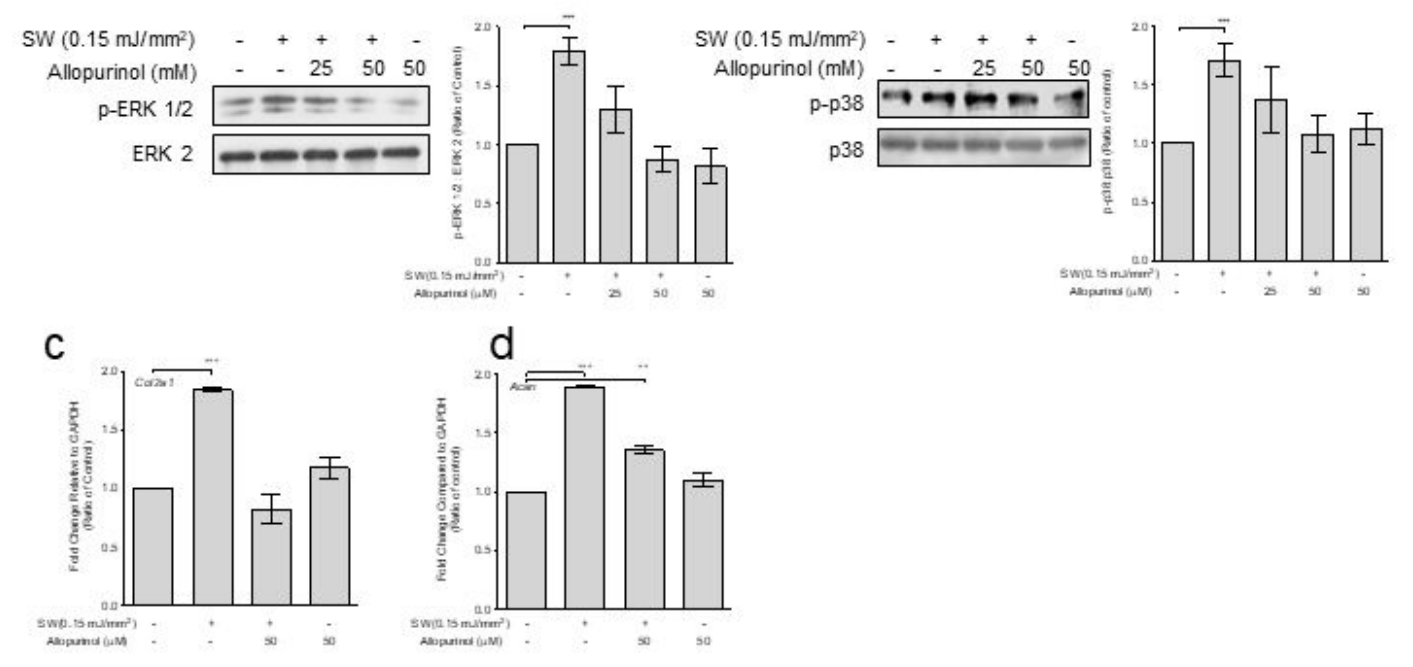

e
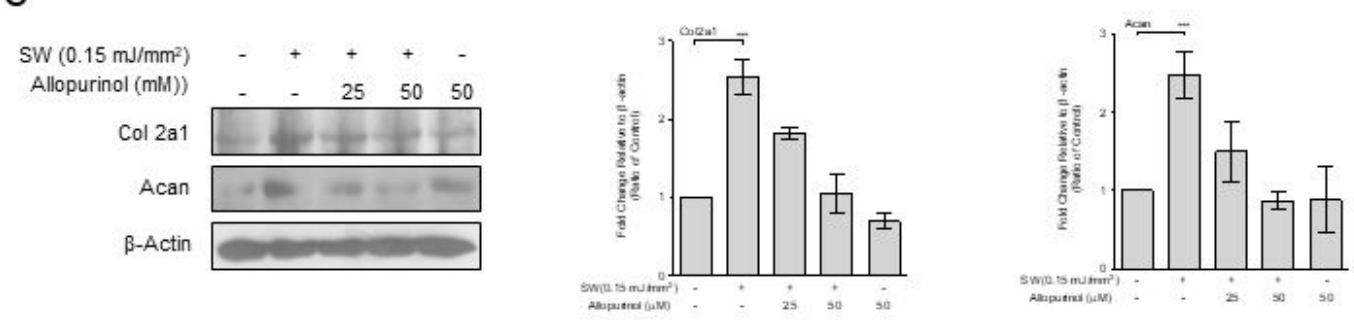

f

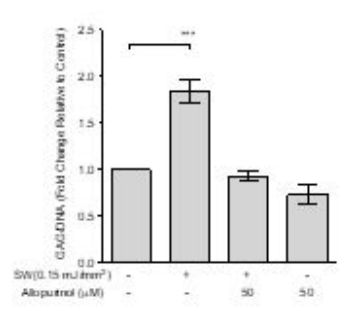

g
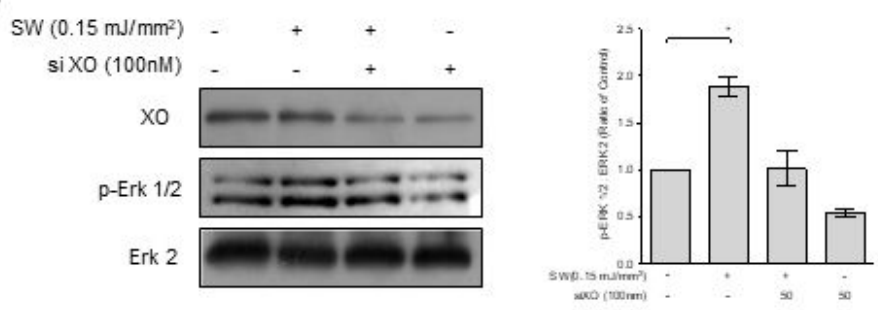

h

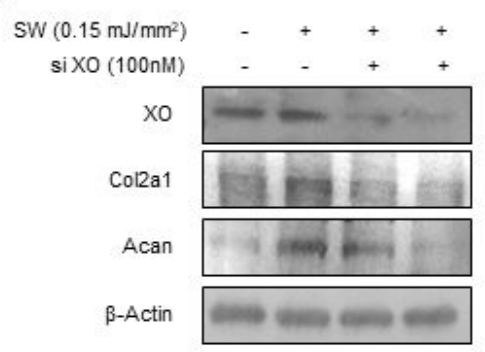

b 
shockwave treatment (500 impulses at $0.15 \mathrm{~mJ} / \mathrm{mm} 2$ ). The gene expression of Col2a1 and Acan was measured by real-time polymerase chain reaction at $24 \mathrm{~h}$ after shockwaves. e The cell pellets were pretreated with allopurinol ( 25 or $50 \mu \mathrm{M}, 1 \mathrm{~h}$ ) and then subjected to a single course of shockwaves (500 impulses at $0.15 \mathrm{~mJ} / \mathrm{mm} 2$ ). The protein expression of Col2a1 and Acan was measured by Western blotting at $24 \mathrm{~h}$ after shockwaves. $\mathrm{f}$ The cell pellets were first cultured for $5 \mathrm{~d}$, followed by three shockwave treatments (500 impulses at $0.15 \mathrm{~mJ} / \mathrm{mm} 2$ ) in $1 \mathrm{wk}$, and then cultured for an additional $7 \mathrm{~d}$ after shockwaves. The cell pellets were pretreated with allopurinol $(50 \mu \mathrm{M}, 1 \mathrm{~h})$ before each course of shockwaves. The cell pellets and all the culture supernatants were collected for the measurement of total sulfated GAG content. g-i Genetic ablation of xanthine oxidase (XO) using specific siRNA abrogated the ROS production after shockwaves in porcine chondrocytes. Chondrocytes were transfected with siRNA specific for XO (100 nM) using nucleofection. Forty-eight hours after the transfections, chondrocyte pellets were treated with a single course of shockwave treatment (500 impulses at $0.15 \mathrm{~mJ} / \mathrm{mm} 2$ ). g-h The protein expression of XO, phosphorylated Erk, total Erk, Col2a1, and Acan. i The amount of total sulfated GAG content. (Each bar represents mean \pm SEM, *: $p<.05 ; * \star p<.01 ; * \star *$ : $p<.001$; Ctrl: Control group; SW: shockwave treatment)

\section{a}
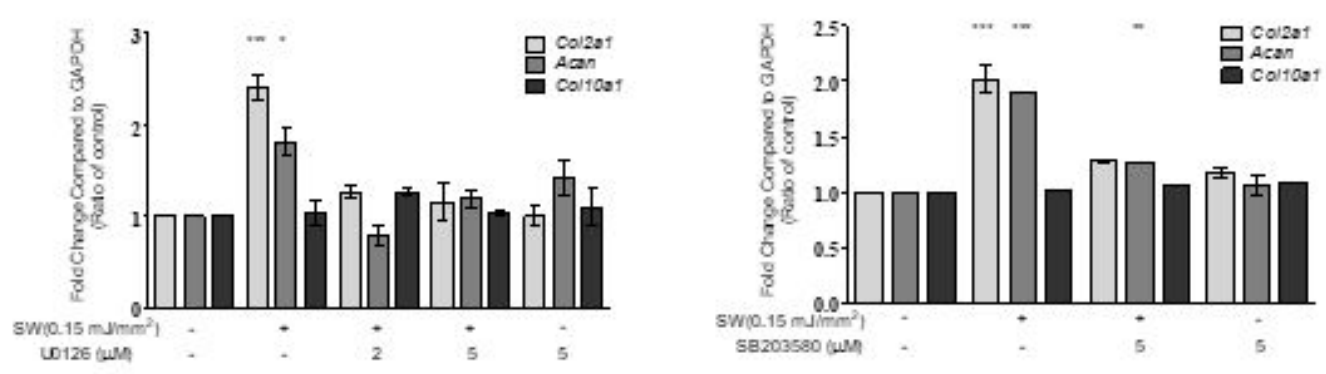

C
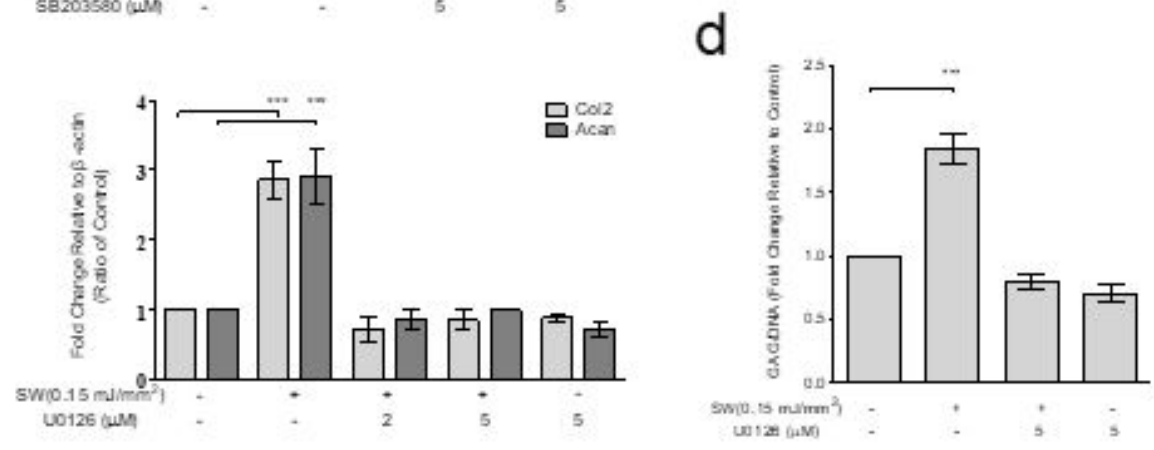

\section{Figure 4}

Shockwaves increased extracellular matrix synthesis of chondrocytes through the activation of Erk $1 / 2$ and p38 signaling. The porcine chondrocyte cell pellets were pretreated with $\mathrm{U} 0126(2$ or $5 \mu \mathrm{M}, 1 \mathrm{~h})$ or SB203580 $(5 \mu \mathrm{M}, 1 \mathrm{~h})$ and then subjected to a single course of shockwave treatment (500 impulses at $0.15 \mathrm{~mJ} / \mathrm{mm} 2$ ). $a-b$ The gene expression of Col2a1, Acan, and Col10a1 was measured by real-time polymerase chain reaction at $24 \mathrm{~h}$ after shockwave treatment. c The protein expression of Col2a1 and 
Acan was measured by Western blotting at $24 \mathrm{~h}$ after shockwave treatment. $\mathrm{d}$ The cell pellets were pretreated with $\mathrm{U} 0126(5 \mu \mathrm{M}, 1 \mathrm{~h})$ before shockwave treatment. The cell pellets and all the culture supernatants were collected for the measurement of total sulfated glycosaminoglycan content using the 1,9-dimethylmethylene blue method. (Each bar represents mean \pm SEM, $*$ : $<<.05 ; * *$ : $p<.01 ; * \star \star: p<.001$; Ctrl: Control group; SW: shockwave treatment)

a
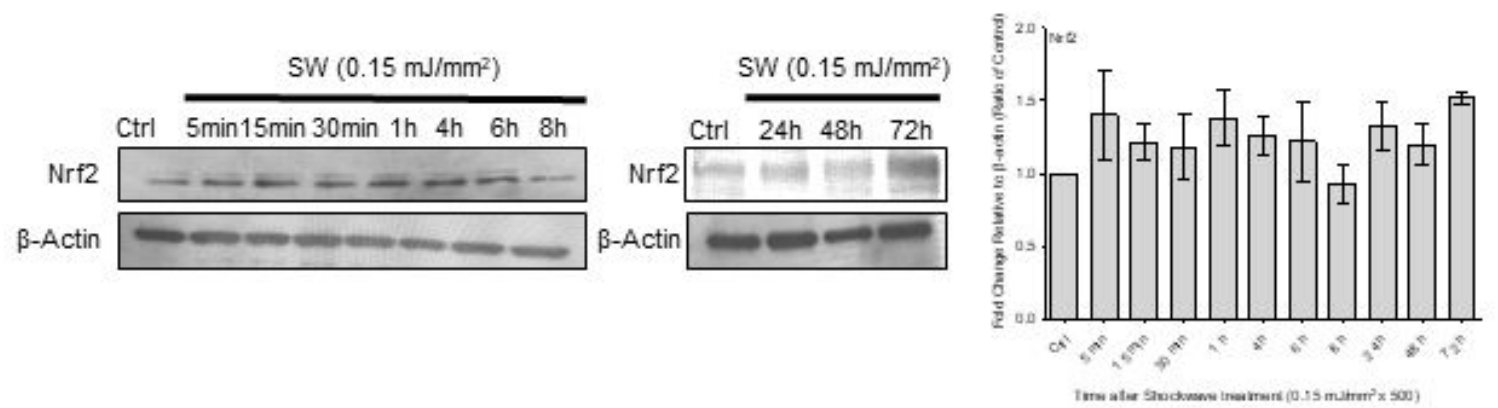

b
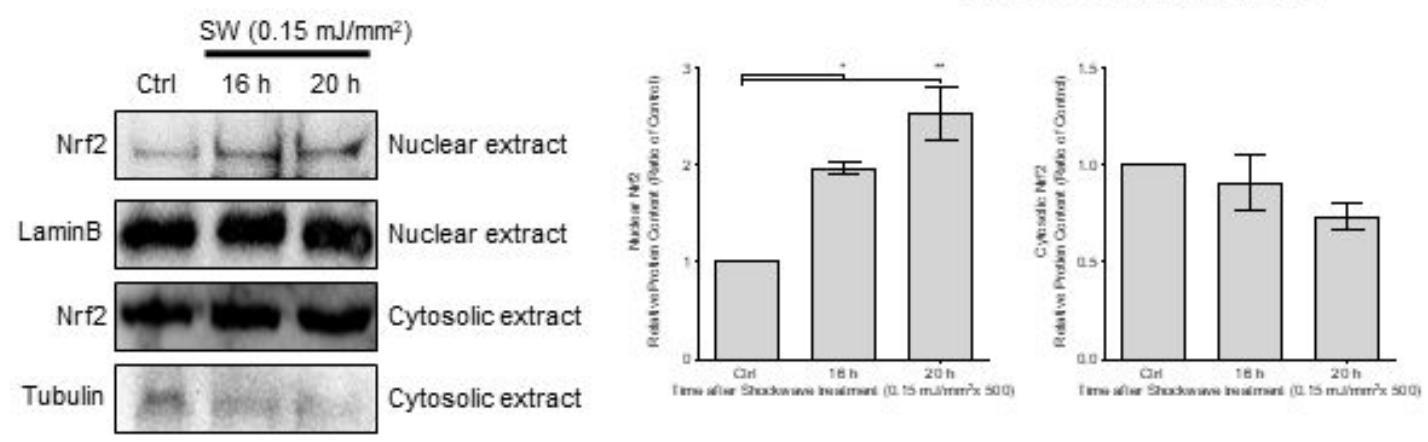

C

d

e

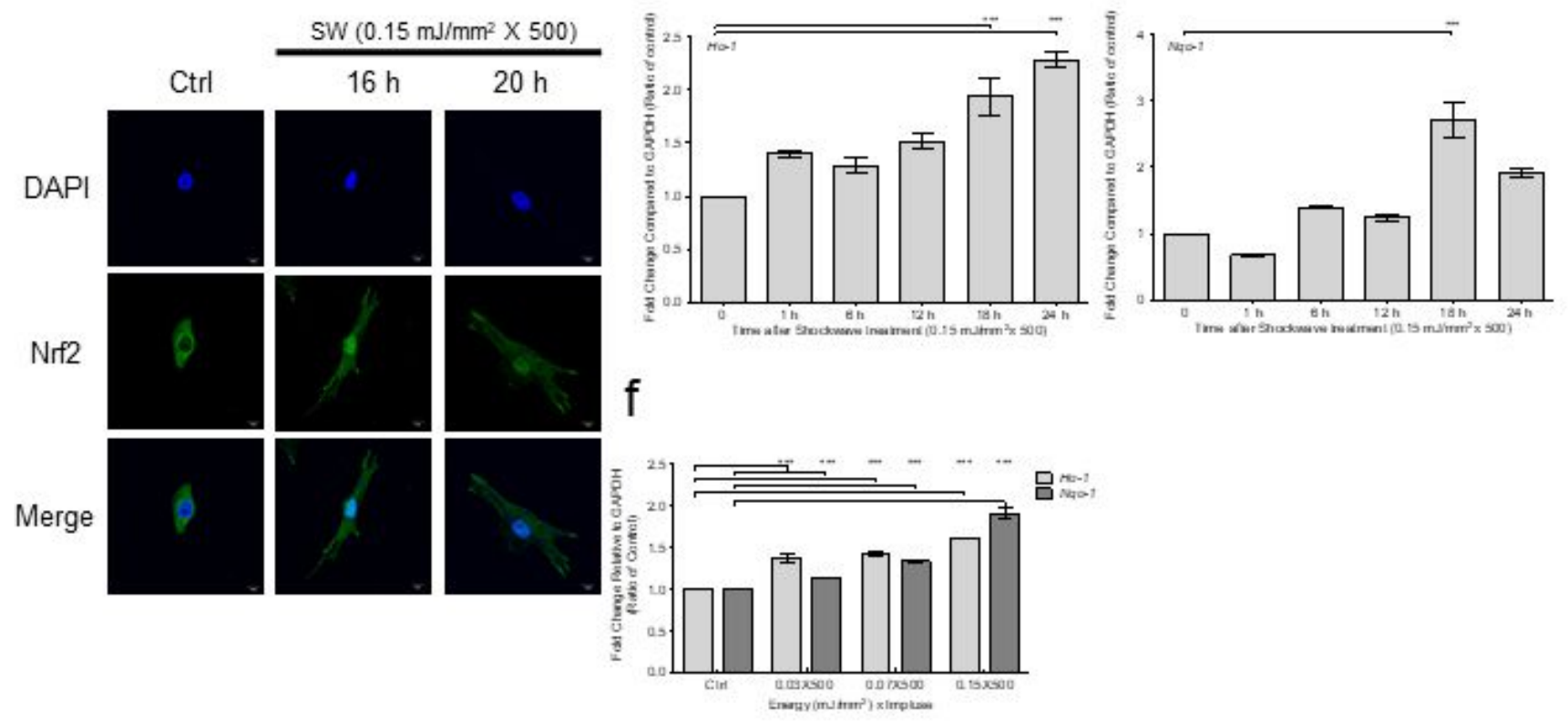

Figure 5 
Shockwave treatment increased the nuclear translocation of Nrf2 and Nrf2-dependent gene expression in porcine chondrocytes. Porcine chondrocyte cell pellets were treated with a single course of shockwave treatment (500 impulses at $0.15 \mathrm{~mJ} / \mathrm{mm} 2$ ). a Protein expression of $\mathrm{Nrf2}$ at indicated time points was investigated by immunoblotting using antibodies against indicated protein. $\beta$-Actin was used as a control for equal loading. The ratio of Nrf2 to $\beta$-actin was calculated by densitometry in each sample, and a value of 1 was given to the control samples. $b$ Nuclear translocation of Nrf2 was measured by Western blotting. Nuclear and cytoplasmic fractions for indicated times after shockwave treatment were purified as described in the Material and Methods section. Tubulin and lamin B were used as loading controls and to specify nuclear versus cytoplasmic expression, respectively. c Nuclear translocation of Nrf2 was investigated by immunofluorescence using specific antibody against Nrf2 and combined with 4',6diamidino-2-phenylindole staining for nuclei. d-e The gene expression of Ho-1 and Nqo-1 at the indicated time points after single shockwave treatment ( 500 impulses at $0.15 \mathrm{~mJ} / \mathrm{mm} 2)$ was measured by realtime polymerase chain reaction (RT-PCR) and was normalized to glyceraldehyde 3-phosphate dehydrogenase (GAPDH). $\mathrm{f}$ The gene expression of Ho-1 and Nqo-1 at $18 \mathrm{~h}$ after shockwave treatments at different dosages was measured by RT-PCR and normalized to GAPDH. (Each bar represents mean \pm SEM, *: $p<.05 ; * *: p<.01 ; * \star *: p<.001 ;$ Ctrl: Control group; SW: shockwave treatment) 


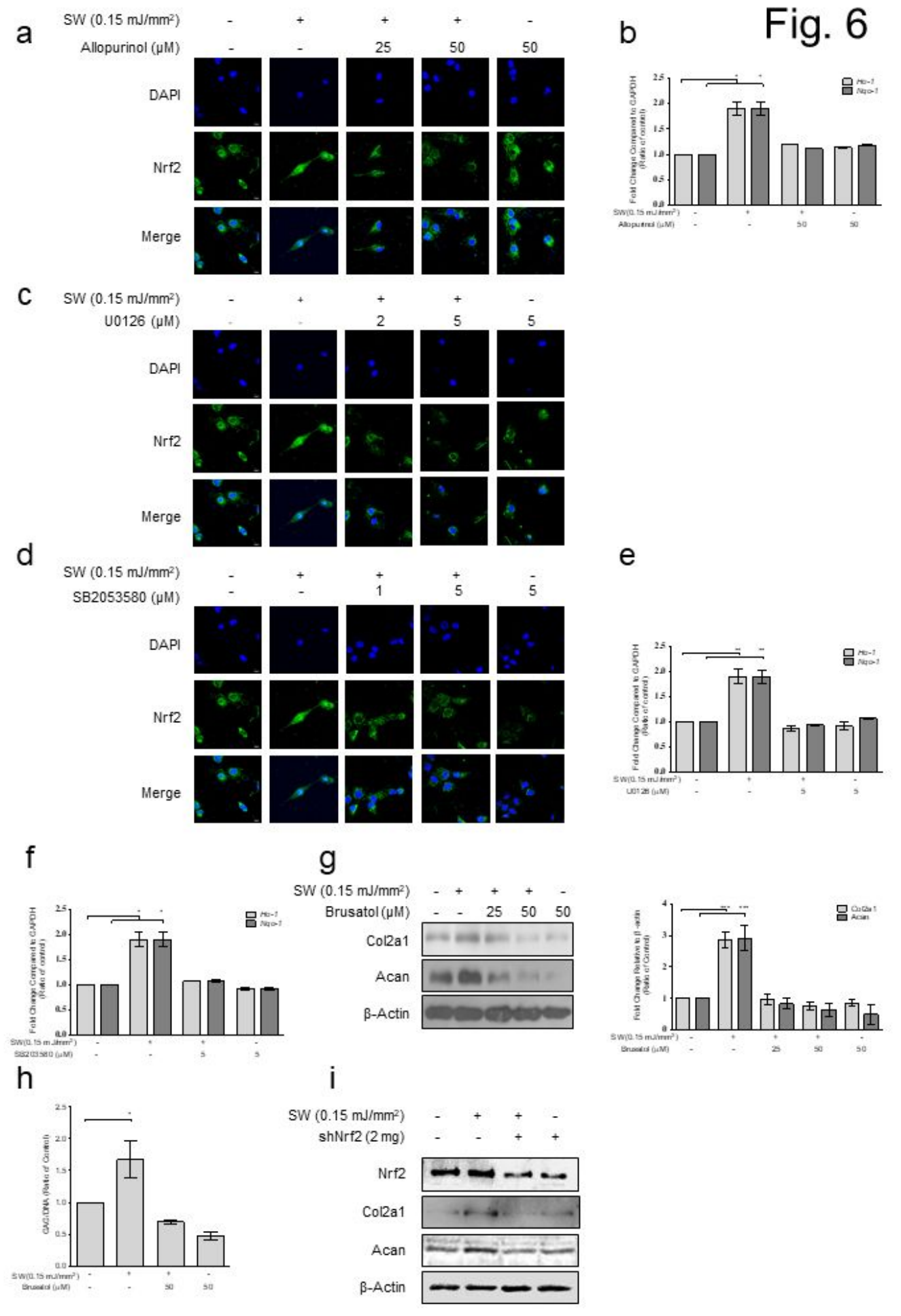

\section{Figure 6}

Shockwaves upregulated Nrf2 activity through ROS and MAPK signaling. Inhibition of Nrf2 abrogated shockwave-increased extracellular matrix synthesis. The porcine chondrocyte cell pellets were pretreated with allopurinol $(25 \mu \mathrm{M}$ or $50 \mu \mathrm{M}, 1 \mathrm{~h}), \mathrm{U} 0126(5 \mu \mathrm{M}, 1 \mathrm{~h})$, or SB203580 $(5 \mu \mathrm{M}, 1 \mathrm{~h})$ and then subjected to a single course of shockwave treatment (500 impulses at $0.15 \mathrm{~mJ} / \mathrm{mm} 2$ ). a, c-d Shockwave-induced nuclear translocation of Nrf2 was investigated by immunofluorescence using specific antibody against 
Nrf2 and combined with 4',6-diamidino-2-phenylindole staining for nuclei. b, e-f The gene expression of Ho-1 and Nqo-1 in pretreated pellets was measured by real-time polymerase chain reaction at $18 \mathrm{~h}$ after shockwave treatment. g The porcine chondrocyte cell pellets were pretreated with brusatol $(25 \mu \mathrm{M}$ or 50 $\mu \mathrm{M}, 1 \mathrm{~h}$ ) and then subjected to a single course of shockwave treatment (500 impulses at $0.15 \mathrm{~mJ} / \mathrm{mm} 2$ ). The protein expression of Col2a 1 and Acan was measured by Western blotting at $24 \mathrm{~h}$ after shockwave treatment. $\mathrm{h}$ The cell pellets were pretreated with brusatol $(25 \mu \mathrm{M}, 1 \mathrm{~h})$ before shockwave treatment. The cell pellets and all the culture supernatants were collected for measuring total sulfated glycosaminoglycan content using the 1,9-dimethylmethylene blue method. i Nrf2 was knocked down through Nrf2-specific shNrf2-expressing lentiviral plasmid. The protein expression of Nrf2, Col2a1, and Acan was measured by Western blotting at $24 \mathrm{~h}$ after a single shockwave treatment ( 500 impulses at $0.15 \mathrm{~mJ} / \mathrm{mm} 2$ ). (Each bar represents mean \pm SEM, *: $p<.05 ; * \star: p<.01 ; * \star \star: p<.001 ;$ Ctrl: Control group; SW: shockwave treatment)

a

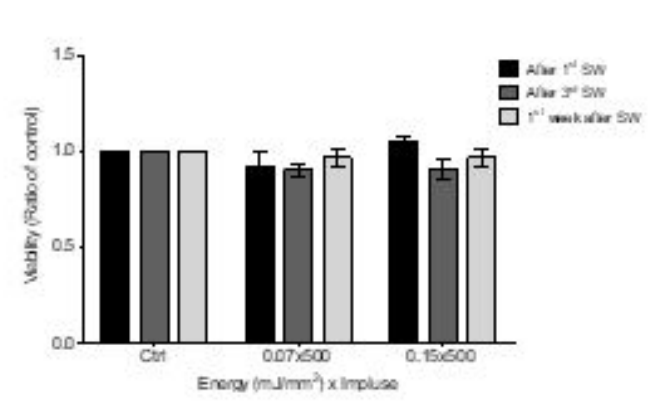

b

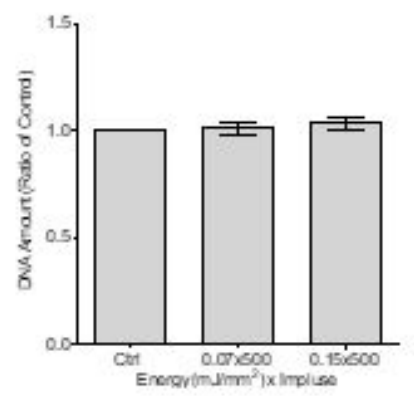

C

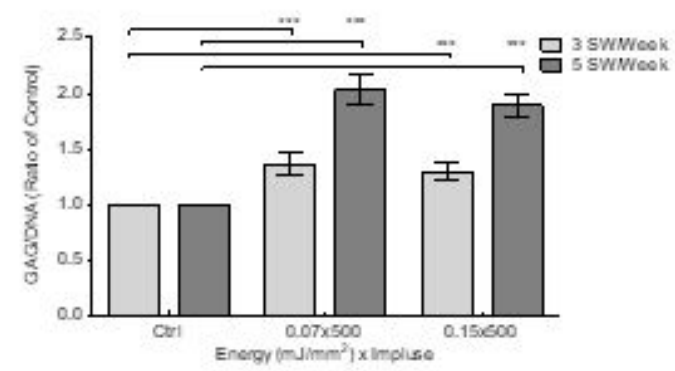

\section{Figure 7}

Shockwaves increased the extracellular matrix synthesis of human OA chondrocytes without affecting cell viability. Human OA chondrocytes were formed in pellets and subjected to shockwave treatment as in the porcine articular chondrocyte experiments described. a Cell viability was investigated by alamarBlue assay after first and third shockwave treatments and $7 \mathrm{~d}$ after the latest treatment. $\mathrm{b}$ The total DNA content of cell pellets was measured by Hoechst DNA assay at $7 \mathrm{~d}$ after the latest treatment. $c$ The cell pellets and all the culture supernatants were collected for measurement of total sulfated glycosaminoglycan (GAG) content using the 1,9-dimethylmethylene blue (DMMB) method. The GAG values were normalized by the DNA content of each sample. (Each bar represents mean \pm SEM, $*: p<.05$; $\star *: p<.01 ; * \star *: p<.001 ;$ Ctrl: Control group; SW: shockwave treatment) 


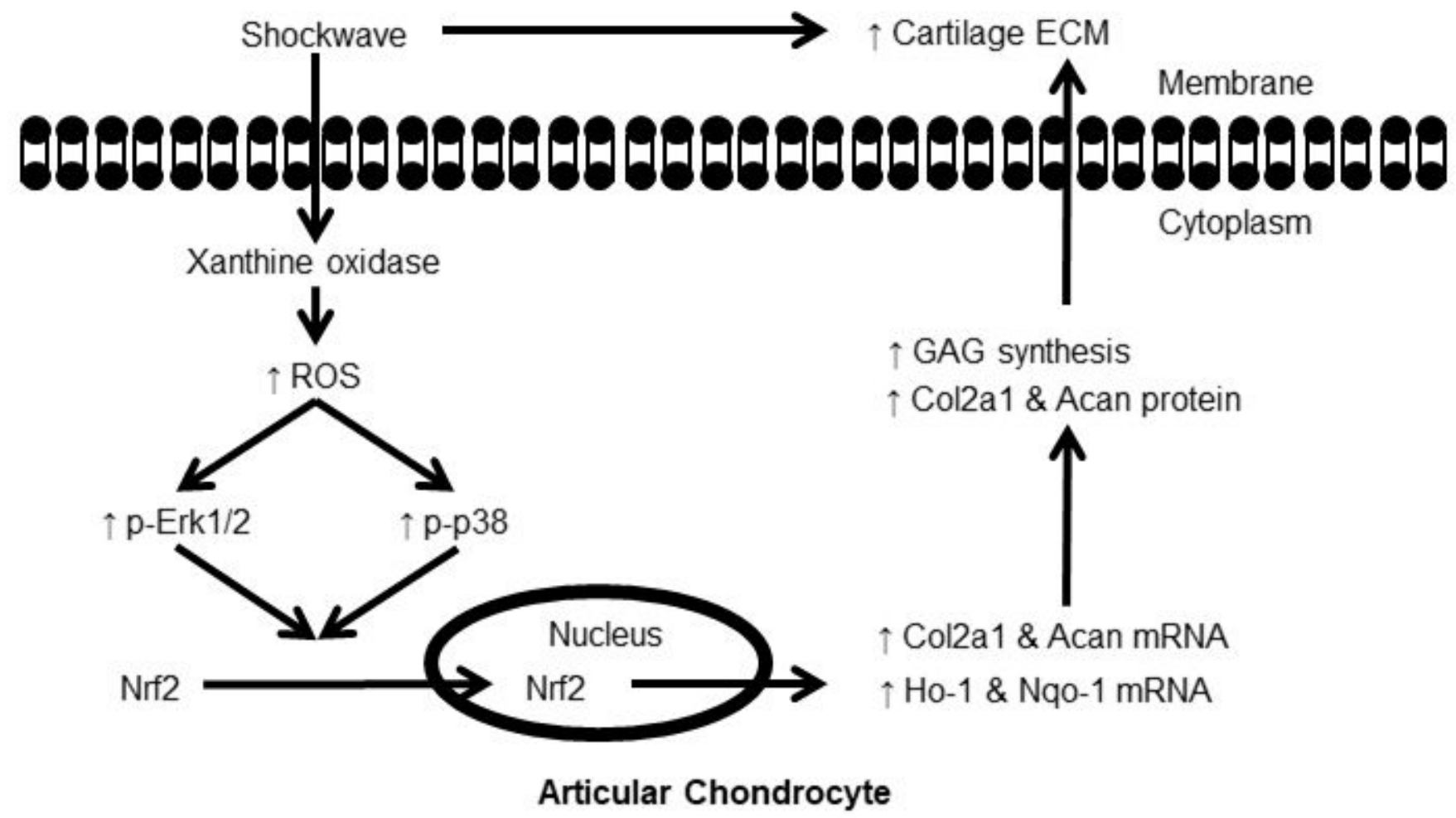

Figure 8

Schematic representation of proposed shockwave mechanism on the increment of extracellular matrix synthesis in chondrocytes. Shockwaves induced the production of reactive oxygen species (ROS) mainly from xanthine oxidase in porcine articular chondrocytes. The low and transient ROS signal activated the phosphorylation of Erk1/2 and p38, which increased the nuclear translocation of Nrf2 and the downstream gene expression of Ho-1 and Nqo-1. Activation of Nrf2 function led to increased extracellular matrix production in porcine articular chondrocytes. 